Portland State University

PDXScholar

$1-1-1985$

\title{
Studies of nucleation and heat transfer in liquid helium isotopes 3 and 4
}

David Lezak

Portland State University

Follow this and additional works at: https://pdxscholar.library.pdx.edu/open_access_etds Let us know how access to this document benefits you.

\section{Recommended Citation}

Lezak, David, "Studies of nucleation and heat transfer in liquid helium isotopes 3 and 4" (1985).

Dissertations and Theses. Paper 385.

https://doi.org/10.15760/etd.385

This Dissertation is brought to you for free and open access. It has been accepted for inclusion in Dissertations and Theses by an authorized administrator of PDXScholar. Please contact us if we can make this document more accessible: pdxscholar@pdx.edu. 
STUDIES OF NUCLEATION AND HEAT TRANSFER

IN LIQUID HELIUM ISOTOPES 3 AND 4

by

DAVID LEZAK

A dissertation submitted in partial fulfillment of the requirements for the degree of

\author{
DOCTOR OF PHILOSOPHY \\ in
}

ENVIRONMENTAL SCIENCES AND RESOURCES-PHYSICS

Portland State University

1985 
TO THE OFFICE OF GRADUATE STUDIES AND RESEARCH:

The members of the Committee approve the dissertation of David Lezak Presented May 24, 1985.

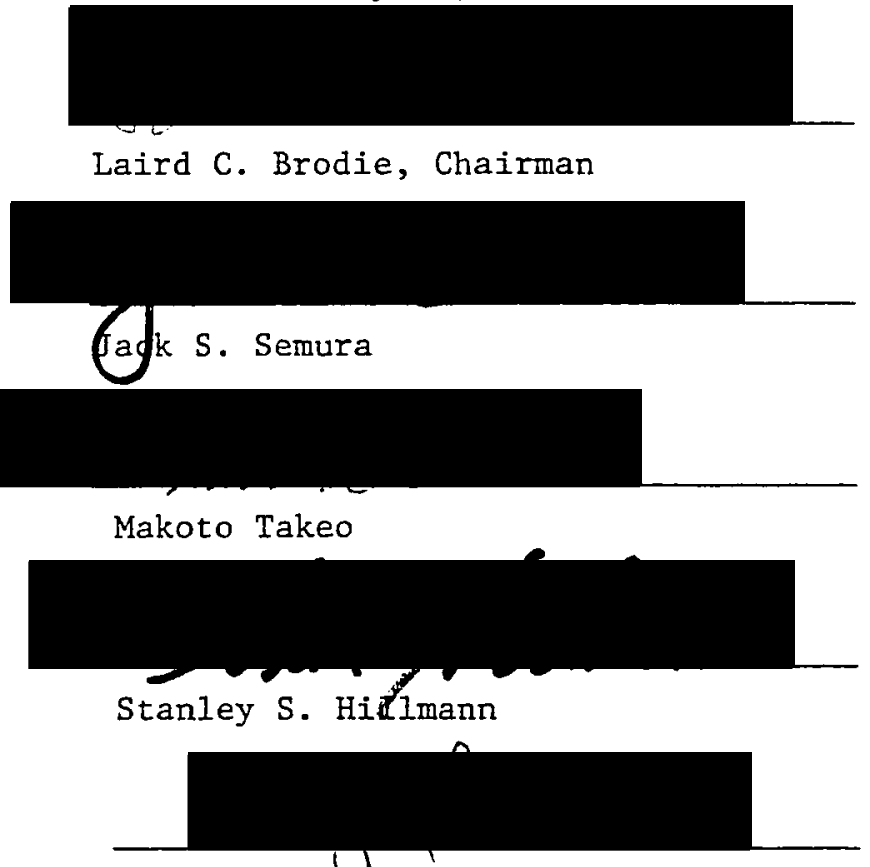

Robert J. O'Btien

APPROVED:

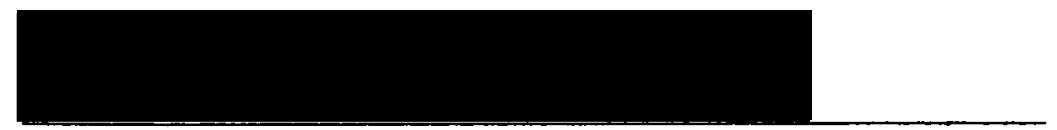

Richard Peterson, Acting Director, ESR

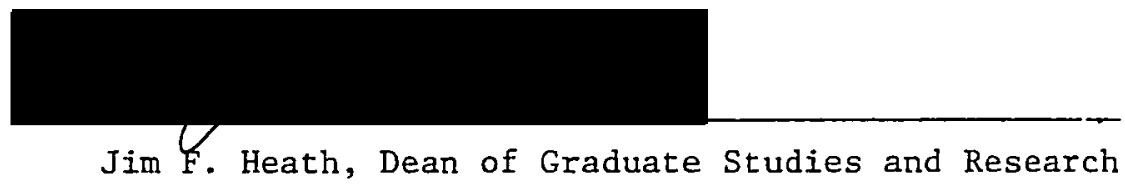


AN ABSTRACT OF THE DISSERTATION OF David Lezak for the Doctor of Philosophy in Environmental Sciences and Resources/Physics presented May 31, 1985.

Title: Studies of Nucleation and Heat Transfer in Liquid Helium Isotopes 3 and 4 .

\section{APPROVED BY MEMBERS OF THE DISSERTATION COMMITTEE:}

Laird C. Brodie, Chairman
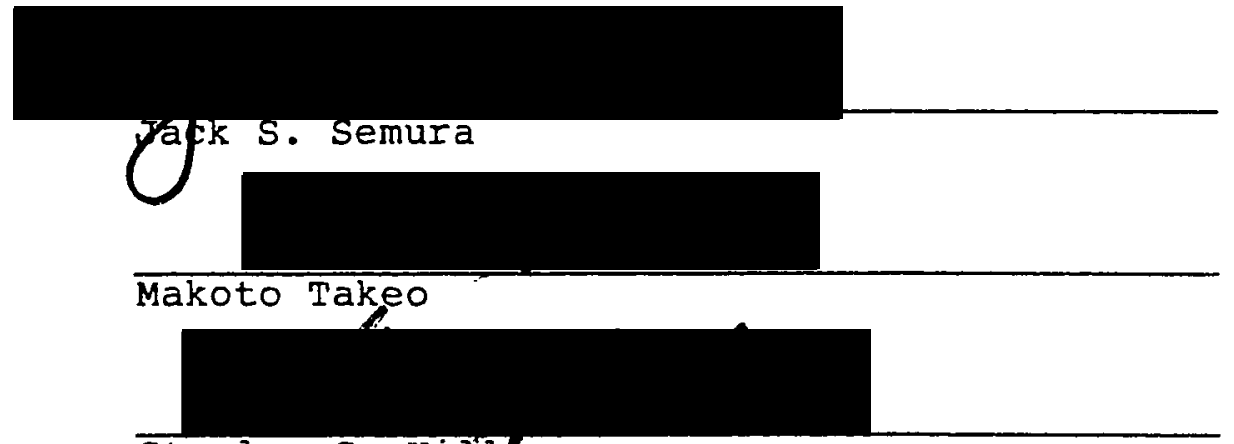

Stanley S. Hillínan

Robert J. O'Bfien

We report the results of a series of experiments in three interrelated areas: 1 ight induced nucleation of vapor bubbles in superheated liquid helium 4, transient heat transfer in liquid helium isotopes 3 and 4 , and homogeneous nucleation of vapor bubbles in superheated liquid helium 3 . This work has resulted in significant contributions in each 
of these particular areas. Our transient heat transfer work has resulted in extremely high temperature measurements of the Kapitza thermal boundary conductance limits in helium 3 and 4 , in measurements of the delay time to the onset of film boiling over a wide range of bath temperatures in helium 4 , and in a determination of bubble growth rates in helium 4. These measurements have been compared with theory and have in some cases allowed the extension or elucidation of that theory. We have characterized the so called "light effect" and established photographically that small amounts of visible light will cause the formation of vapor bubbles at the interface of a solid and superheated liquid helium 4 and that this vapor can influence the quasi-steady-state heat flux vs temperature hysteresis curve. Finally, we have measured the homogeneous nucleation temperature of liquid helium 3 and found good agreement with the predictions of the Becker-Doring-Volmer-zel'dovich-Frenkel nucleation theory. This work is shown to have applications to practical cryogenic engineering, to further understanding of basic heat transfer and nucleation theory, and to practical and theoretical environmental and resource considerations. 


\section{ACKNOWLEDGEMENTS}

Progress in science typically results from a group endeavour with more hours spent discussing ideas, concocting theories, planning experiments, and proofreading and criticising papers than are spent in the direct scientific activities of experimental or theoretical investigation. The work discussed in this dissertation is no exception and would not have occurred without the assistance of Drs. Jack Semura and Laird Brodie. I owe them a great debt for their willingness to bring me into their research group.

Prior to my arrival, and for a few overlapping years, Dr. Dipen Sinha was the principal experimentalist and senior graduate student in the lab. In his zealous pursuit of knowledge he blazed a trail whose various meanderings I am still exploring. Because of his habit of dog-earing the pages of journal articles that he found particularly interesting, my sense of rediscovery was more real than might otherwise be imagined. In some way or another, Dr. Sinha's work has formed the basis of almost everything reforted here.

Much of my experimental work involved the manipulation of the flow of electrons through pieces impure silicon. What little competence in this art that I now possess must 
be due to the never ending tutelage of Brian McLaughlin. His excellence as a teacher is sometimes overshadowed by his skill as a technician but it is appreciated by generations of graduate students like myself. Lee Thannum, Rudi zupan, and Garo Arakelian, also of the Science shop, have been helpful beyond words for these last few years. In addition, I am greatly indebted for the understanding and guidance that I have received from Drs. Mark Gurevitch and Ray Sommerfeldt and from Mrs. Dawn Dressler.

Finally, let me again thank Dr. Laird Brodie, although this time for acting as my mentor and thesis advisor. I consider myself almost uniquely fortunate to have had the opportunity to work with as dedicated a teacher and researcher as he is. There are lessons that I have learned by his patient examples that I hope never to forget. 
TABLE OF CONTENTS

PAGE

ACKNOWLEDGMENTS ...................... ii

IIST OF TABLES....................... viii

IIST OF FIGURES..................... ix

CHAPTER

I INTRODUCTION...................

Light Induced Nucleation

Transient Heat Transfer

Homogeneous Nucleation in ${ }^{3} \mathrm{He}$

I I LIGHT INDUCED NUCLEATION.............

Quantification of the Light Effect..... 8

Factors Affecting the Magnitude of

the Light Effect..............

12

Photographic Studies of Light Induced

Nucleation of Boiling at the Inter-

face of a Heated Solid and

Superheated Liquid Helium I.......

Photographic Observations

Test of the Photoelectron Hypothesis...

Discussion

Steady State Effects.............. 
Applications of the Light Effect......

Measurement of the Kapitza Thermal Boundary Conductance from Bismuth to Normal Liquid Helium Isotopes 3 and 4 at Their Homogeneous Nucleation Temperatures.............

Transient Solid-Liquid Helium Heat Transfer and the Time Delay in the onset of Film Boiling.......... 35

Background

Theoretical Background

Experimental..................

Effect of Surface Tension

Observations

Error Analysis

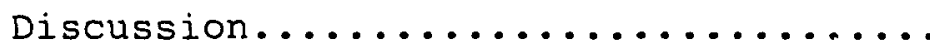

REFERENCES.......................

APPENDIX A

Growth of Single Bismuth Crystals for Magnetoresistive Thermometry in Liquid Helium. 
APPENDIX B

Use of Germanium Crystals and Rhodium-

Iron Wire for Fast Transient Thermometry

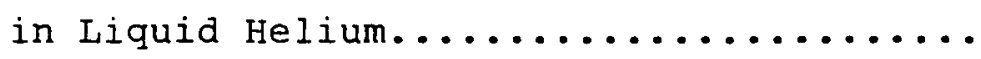

APPENDIX C

Crystal Balance Techniques for Fast

Transient Thermometry Using Non-ohmic

Resistive sensors................

82 


\section{LIST OF TABLES}

TABLE

PAGE

I A Comparison of the Properties of Bismuth, Riodium/Iron, and Germanium with Reference to Their Suitability to Fast Transient Thermometry in Liquid Helium Isotopes 3 and $4 . . . . .81$ 


\section{LIST OF FIGURES}

FIGURE

PAGE

1. Effect of Pulsed Light on Superheat vs Time

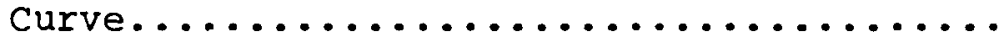

2. Effect of Pulsed Light on Superheat vs Time

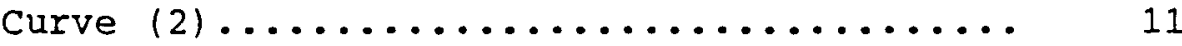

3. Light Effect vs Bath Temperature......... 13

4. Light Effect vs Light Wavelength......... 15

5. Light Effect Photograph............... 17

6. Quasi steady-state q vs $\Delta^{\mathrm{T}} \ldots \ldots \ldots \ldots \ldots \ldots \ldots . . \ldots 22$

7. Film Thickness vs Time After Light Flash... 26

8. Superheat vs Heat Flux.............. 32

9. Superheat vs Time for Film Boiling........ 38

10. Delay Time vs Heat Flux.............. 40

11. Delay Time vs Bath Temperature......... 45

12. Delay Time vs Bath Temperature (2) ....... 46

13. Scaled Homogeneous Nucleation Temperature vs Scaled Pressure................. 50

14. Experimental Apparatus for ${ }^{3} \mathrm{He} \ldots . . . . . .59$

15. Superheat vs Heat Flux............... 62

16. Scaled Homogeneous Nucleation Temperature

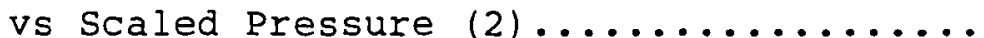


FIGURE PAGE

17. Schematic Circuit Diagram.............. 84

18. Typical superheat vs Time Showing

Electrical Transients.................. 86

19. a) Balance Curve for a Crystal without

Non-ohmic Behaviour. b) Balance Curve for

a Crystal with Non-ohmic Behaviour........ 
CHAPTER I

INTRODUCTION

This dissertation describes the results of a series of experiments in three interrelated areas: 1 ight induced nucleation of vapor bubbles in superheated liquid helium 4, transient heat transfer in liquid helium isotopes 3 and 4 , and homogeneous nucleation of vapor bubbles in superheated liquid helium 3. In addition, there is a set of appendices describing several particular experimental aspects of the work reported here that were not deemed to be in and of themselves appropriate for publication.

Because experimental and theoretical work in a broad range of areas is included in this dissertation, it is difficult to single out any one contribution as more significant than the others. As discussed in particular below, the results of our work in each of the three major areas have application to both theoretical and applied physics, as well as to practical cryogenic engineering.

The main body of this dissertation is divided into three chapters each concentrating on one of three interrelated areas of research. The first major chapter of this dissertation is on the area of light induced nucleation of vapor bubbles in superheated normal 1 iquid helium 4 and the 
enhancements to transient and steady state heat transfer that result. This research is based on the observation that ordinary room light shining through the unsilvered glass walls of our cryomagnetic dewar influenced the shape of the $\Delta^{T}$ vs time curves in a transient superheating experiment. We have found that this "light effec:." occurs at any superheated liquid helium-solid interface, whether the solid be a metal, semi-metal, or semiconductor, and that the effect is strongly dependent on the light wavelength and bath temperature along the vapor pressure coexistence curve. In those circumstances where it is strongest, the light effect was the single most sensitive detector of near UV radiation in our laboratory and was able to induce the formation of vapor much more suddenly than even our highest power joule heating experiments. We have shown that an understanding of the light effect could be of importance in practical cryogenic engineering applications, in experimental studies of heat transfer and bubble growth mechanisms in liquid helium, and in the acquisition of fundamental knowledge about the process of nucleation in general and the influence of visible light on that process in particular.

The second major chapter of this dissertation deals with heat transfer mechanisms in Iiquid helium 3 and in normal and superfiuid liquid helium 4. We have studied the time delay to the onset of film boiling in normal liquid helium 4 and have found that this delay can be predicted by 
a relatively simple equation which is based on a few elementary assumptions about the limiting physical and hydrodynamic factors in the development of a stable film boiling configuration. These results are likely to be of relevance to any application in which liquid helium is used as a high efficiency coolant and of particular importance to superconductor applications where temperature transients are to be expected and the ability to stabilize these excursions must be included in the basic design. We have also measured the Kapitza thermal boundary conductance limits in normal and superfluid liquid helium 4 and in helium 3 . Our measurements in the normal liquids were made using the homogeneous nucleation temperature as a stable reference point, rather than a large superfluid bath as has previously been the custom. This has allowed the extension of these measurements to higher temperatures.by almost a factor of two. Kapitza conductance and phonon radiation phenomena in general are becoming increasingly important in the very practical areas of microscopy and materials characterization as well as in extending our fundamental understanding of how non-electromagnetic radiation interacts with matter.

The third major chapter of this dissertation deals with the measurement of the homogeneous nucleation temperature of liquid helium 3. This measurement extends previous work in our laboratory in helium 4 to the very limits of low temperatures and large relative quantum mechanical influence. 
Our results are shown to be in good agreement with the predictions of the Becker-Doring-Volmer-Zel'dovich-Frenkel nucleation theory as well as the quantum extention to the classical corresponding states analysis suggested during the pioneering work in helium 4. The BDVZF nucleation theory provides a common conceptual basis for all nucleation processes that are encountered in our environment through the crystallization of supercooled liquids, condensation of supersaturated vapors, boiling of superheated liquids, and cavitation of stressed liquids so our work in helium 3 has been important in testing and verifying the validity of this theory over as broad a range as possible. In addition, this work forms the basis of a continuing project to achieve quantum tunnelling nucleation--a process that has been predicted to occur under potentially realizable circumstances but has never been observed.

Finally, there are three appendices. The first discusses the growth of bismuth single crystals for magnetoresistive thermometry. The second deals with the adaptation of germanium crystals and rhodium-iron wire to transient heater-thermometer applications, and the third with certain aspects of fast transient thermometry in bismuth, which shows variable but distinct non-ohmic behavior. 
CHAPTER II

LIGHT INDUCED NUCLEATION IN SUPERHEATED LIQUID HELIUM 4

INTRODUCTION

Following the observation that ordinary room lights could influence the shape of the $\Delta^{T}$ vs time curve in a transient superheating experiment in normal Iiquid helium 4 , our laboratory began an investigation of what became known as the "Light Effect." Preliminary studies showed that when the liquid in contact with the surface of a bismuth crystal heater-thermometer had been rapidly superheated to at least a certain threshold temperature, the flashing of a single strobe light pulse of several microseconds duration through the unsilvered sides of a helium glass dewar caused a sudden large drop in crystal superheat (Sinha 1982a). The effect was striking: the temperature of the crystal could decrease by as much as 758 of the total superheat in $3 \mathrm{msec}$, with approximately 908 of the temperature drop occurring in under a millisecond.

Our preliminary hypothesis, later confirmed photographically, was that the sudden increase in heat transfer across the solid-liquid helium interface was caused by an increase in bubble activity and that the light had somehow 
induced the nucleation of bubbles at the interface. The exact mechanism of this nucleation remains unclear; however several hypotheses had been considered including surface heating by the light, mechanical or phonon effects, and photoelectrons. Each of these hypotheses have been seriousIy compromised by experimental results and thus there remains the intriguing question of how exactly visible light can cause the nucleation of vapor bubbles in superheated liquid helium (Sinha 1980, Lezak 1984a).

\section{EXPERIMENTAL}

Our experiment utilizes a method of transient thermometry that exploits the magnetoresistance of single crystals of bismuth. At liquid helium temperatures, this magnetoresistance exhibits a strong temperature dependence which al lows the determination of the temperature of the liquid helium in contact with the crystal surface. A step function electric heating current is applied to the bismuth crystal at time $t=0$. We monitor the subsequent temperature change, $\Delta^{T}(t)$, of the crystal as a function of time by measuring the voltage $\Delta V(t)$ between two potential leads in a 4-terminal network. A voltage proportional to the current $I(t)$ is derived from an external resistance in series with the crystal, and the resistance change $\Delta^{R}(t)$ is obtained by electronically evaluating $\Delta^{V(t) / I(t)}$. Finally, $\Delta^{T}(t)$ is found from a calibration curve of $\Delta^{R}$ vs $\Delta^{T}$. We can heat the 
crystal at any desired rate by varying $I(t)$, so the device serves as a heater as well as a thermometer. In our experiment transient temperature measurement errors arising from uncertainty in Kapitza resistance and other sources are estimated to be less that $50 \mathrm{mk}$. The thermometer response time is less than 100 us so the temperature excursions on a millisecond time scale are easily measured and reproduced. Complete details of the bismuth thermometer and the associated electronic circuitry have been published elsewhere (Brodie 1977, 1978, 1981). The bismuth crystal was mounted in a conventional Pyrex double dewar system with an unsilvered bottom transmitting light of wavelength longer than $310 \mathrm{~nm}$ and fitting between the pole pieces of an electromagnet. We have used a variety of light sources in our experiments including a General Radio type 1538-A Strobotac and a Sunpack Auto-322 photo flash. A variable delay circuit allowed the light to be flashed at any desired time after the application of the step-function heating current. These continuous spectrum Iight sources were normally used in conjunction with a 0.25 meter monochromator with $1 \mathrm{~mm}$ entry and exit slits. Wavelengths of unwanted orders were eliminated from the monochromator output with long wave passband filters. A calibrated photocell was used to monitor the light pulse irradiance over the time of the light flash in order to establish the total light pulse energy. 
QUANTIFICATION OF THE LIGHT EFFECT

Our inital observations of the light effect were based on qualitiative comparisons of temperature vs time curves in transient superheating experiments. A typical observation is shown in Fig. 1 where curve a indicates the transient superheating behavior in the absence of light and curve $\underline{b}$ is due to the superposition of a curve resulting from a flash of light at the time indicated by the arrow. of the many parameters available to quantify the light effect we have elected to use the difference between the slopes of a $\Delta^{T} v s$ time curve resulting from an immediately preceding light flash and a reference curve recorded without light but under otherwise identical conditions. By applying the power balance equation to the heater-thermometer we now show that this difference in slope is proportional to the increased heat flux due to the light. We have:

$$
p=q+(m / a) c_{p}(T)(d T / d t),
$$

where $p$ is the electric power input per unit area, $q$ is the heat flux out of the crystal, $\mathrm{m} / \mathrm{a}$ is the mass per unit surface area of the crystal, $C_{p}(T)$ is the constant pressure heat capacity of the crystal per unit mass, and $\mathrm{T}$ is $\mathrm{T}_{\text {sat }}+$ $\Delta^{T}$. If we analyze the difference between a curve following a light flash and a reference curve we find:

$$
\Delta(p-q)=\left(p-p_{0}\right)-\left(q-q_{0}\right)=(m / a) c_{p}(T)\left[d\left(T-T_{0}\right) / d t\right],
$$

where the subscript zero denotes reference curve quantities. 


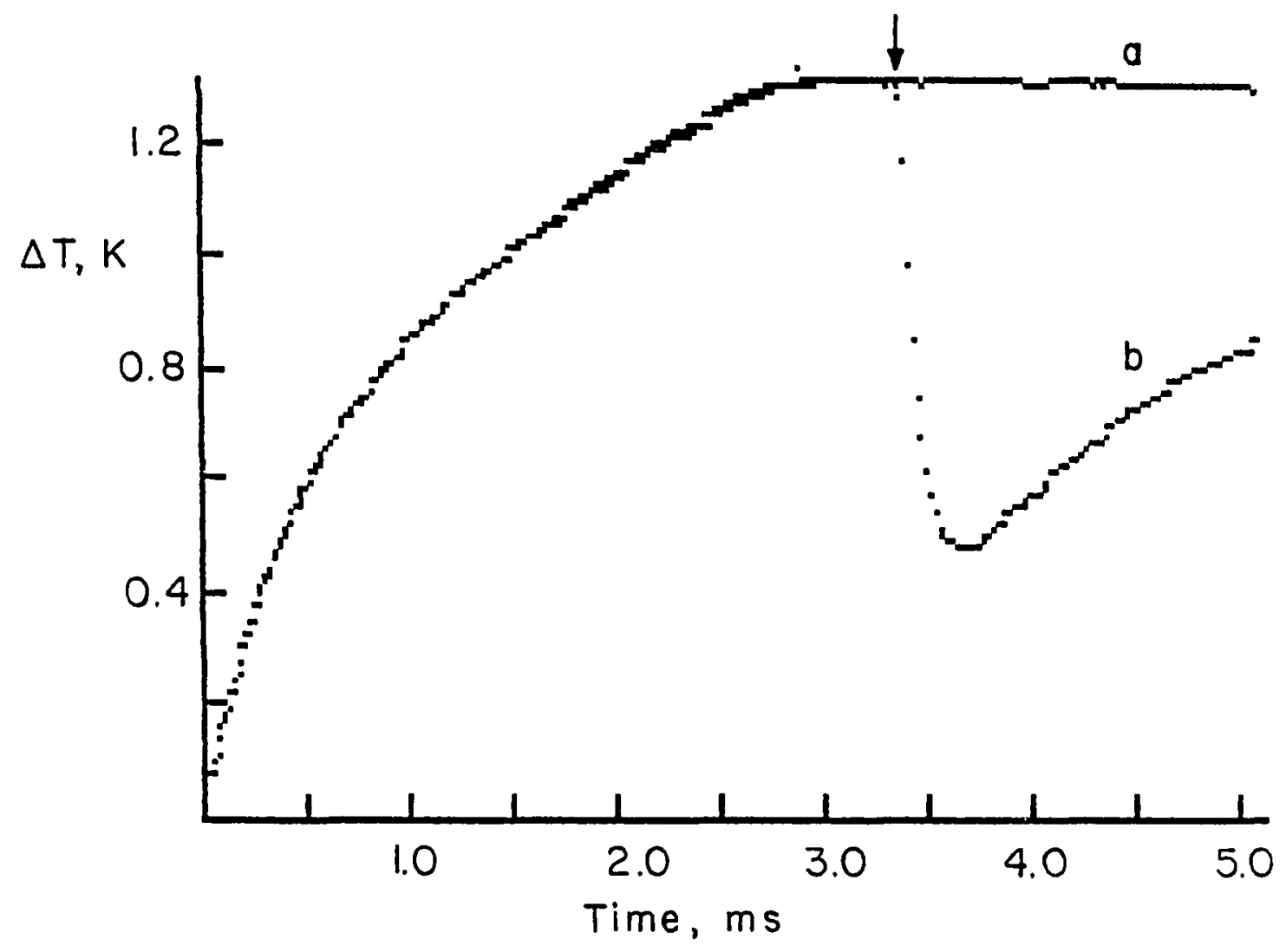

Figure 1: Effect of pulsed light on the superheat $\Delta^{T}$ vs time curve. A light pulse of approximately 5 us duration and irradiance at the crystal surface of 24 $\mathrm{mW} / \mathrm{cm}^{-2}$ is applied at the time indicated by the arrow. Curve a shows the temperature as a function of time for the case in which no light pulse is applied and curve b shows the sharp drop in temperature following the light pulse. 
Because $\mathrm{p} \approx \mathrm{p}_{\mathrm{O}}$ we find:

$$
\Delta q=\left(q-q_{0}\right) \doteq-(m / a) C_{p}(T)\left[d\left(T-T_{0}\right) / d t\right] \text {. }
$$

Thus the change in the first time derivative of the temperature vs time curve is proportional to the additional heat flux out of the crystal resulting from the flash. Since, as shown in Fig. 1, the light was flashed at a time when the $T_{0}$ curve is essentialiy constant, $\mathrm{dT}_{\mathrm{o}} / \mathrm{dt}$ is small enough to be neglected in comparison with dT/dt.

Figure 2 a shows a typical record of the $\Delta^{T}$ vs time curves that we used to calculate the slope, $\mathrm{dT} / \mathrm{dt}$. It is much like the trace of Fig. 1 except that the temperature and time scales have been expanded around the instant of the light flash to optimize the resolution of our digital oscilloscope. This curve, composed of 512 data pairs, was then entered into a microcomputer which computed an average value of $\mathrm{dT} / \mathrm{dt}$ in the interval from 0.1 to $0.4 \mathrm{~ms}$ after the light flash. In the discussion and graphs which follow, this. value will be used as the single quantitiative measure of the light effect. Some experimental traces were also subjected to a higher order polynomial curve fit. Figure 2 $\underline{b}$ shows the result of evaluating the curve of Fig. 2 a by this technique. The data points prior to the light flash were not included in the curve fit program in order to avoid the physically expected discontinuity of slope in the first few tens of microseconds where the light induced cooling begins to develop. We have not used the maximum cooling 


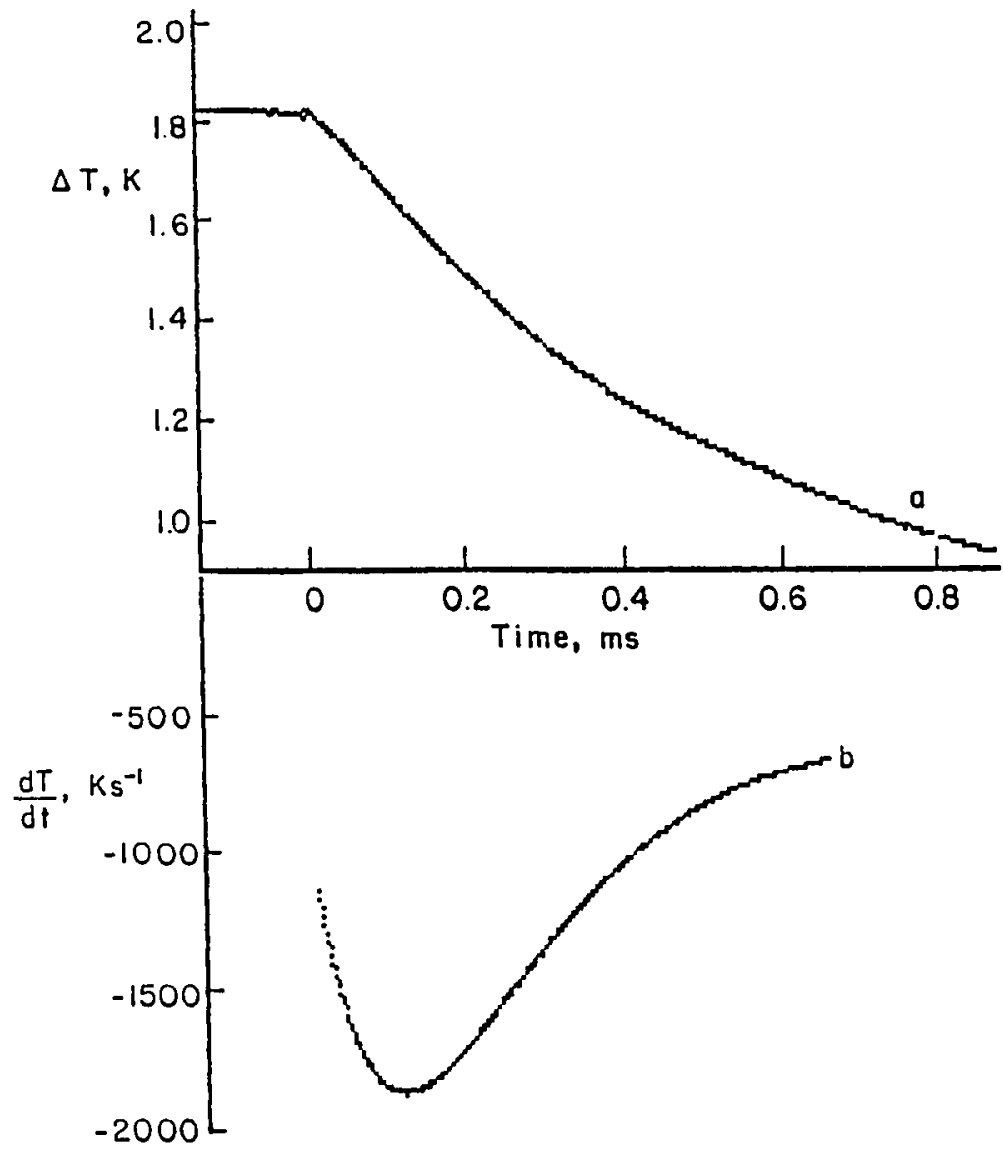

Figure 2: a - Effect of pulsed light on the superheat $\Delta^{\mathrm{T}}$ vs time curve. A light pulse is applied at the time $t=$ 0 . This figure is similar to the one of Fig. 1 except that the temperature and time scales have been expanded around the time of the light flash to improve the resolution. $b-d T / d t$ vs time. This curve is the result of a polynomial least squares fit to the curve of Fig 2a. The peak value of $-1.86 \times 10^{-3} 3 \mathrm{~K} / \mathrm{sec}$ corresponds to an additional heat flux from the crystal into the liquid helium of $520 \mathrm{~mW} / \mathrm{cm}^{-} 2$. 
rate computed by this method to quantify the light effect because of wide variations in the shape of the dT/dt vs time curve under different circumstances and possible limitations due to the finite response time of the thermometer and associated electronic circuitry.

\section{FACTORS AFFECTING THE MAGNITUDE OF THE LIGHT EFFECT}

The magnitude of the light effect, as measured by the method described above, is influenced by a number of factors. We have been most interested in characterizing these influences to examine the nature of the mechanisms involved in the light effect.

As expected, the effect varies with applied light pulse energy. In the range of energies typical of our experiments, we have found slopes in the range 0.4 to 0.5 for loglog plots of light effect vs light pulse energy. This suggests that the l,ight effect varies roughly as the square root of the applied light pulse energy.

The effect is strongly dependent on bath temperature and pressure. As we move down in temperature and pressure along the vapor pressure curve the magnitude of the light effect increases by a factor of about 80 bewteen 4.2 and 2.2 K. This increase, shown in Fig. 3, is roughly exponential with decreasing temperature. We believe that this dependence is due to the increase in both $\Delta^{T}$ and the difference between the Iiquid and vapor density, $\left(\rho_{1}-\rho_{v}\right)$, as the bath 


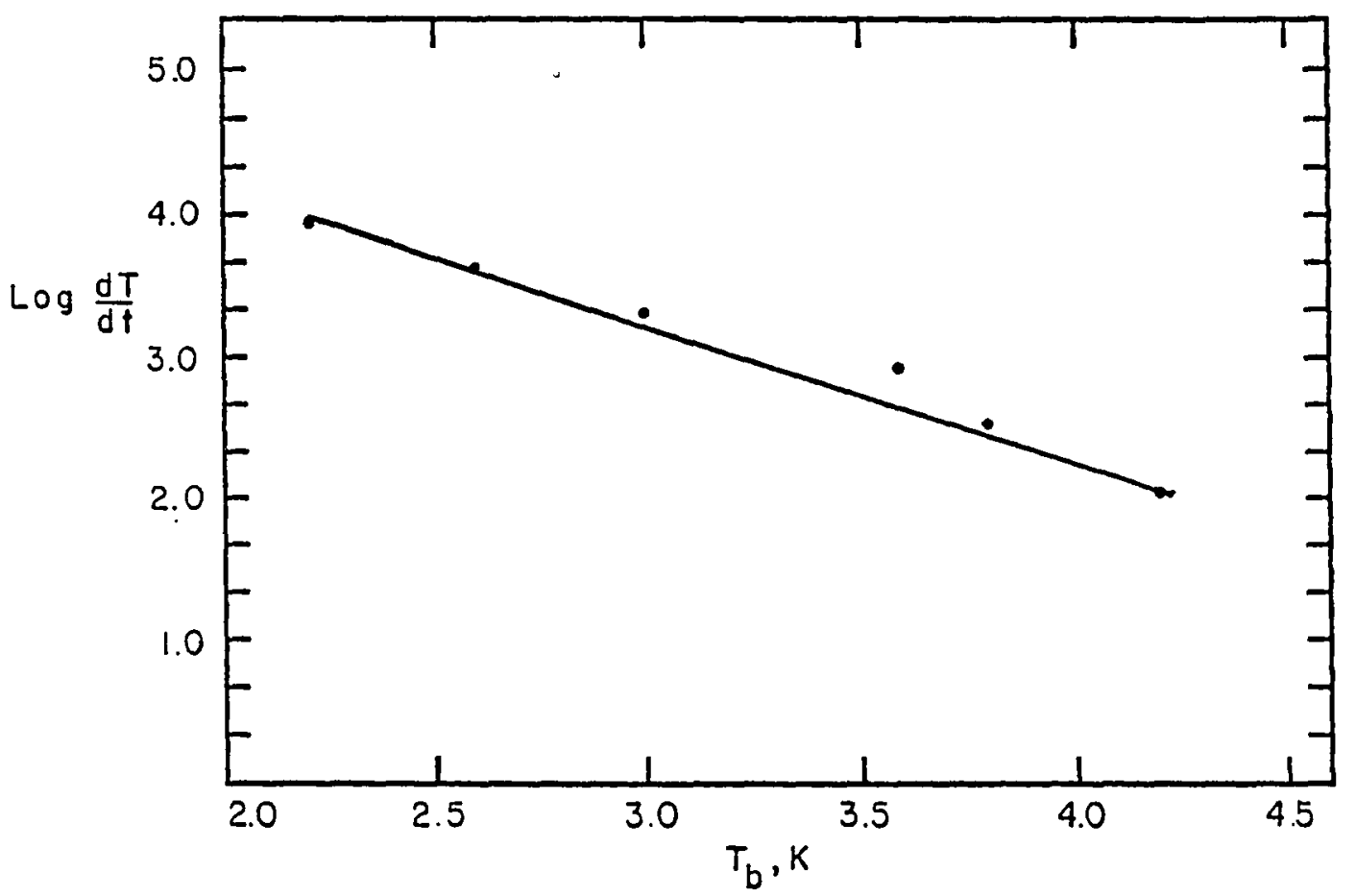

Figure 3: Light effect vs bath temperature. The values of $\mathrm{dT} / \mathrm{dt}$ are obtained by the method described in the text. 
temperature is lowered.

The light effect is also strongly wavelength dependent. Figure 4 shows three typical curves of $\mathrm{dT} / \mathrm{dt}$ as a function of wavelength, $\lambda$. In the interest of clarity we have only shown three of a family of curves that result from repeating the wavelength sweep at various bath temperatures and/or light pulse energies. For any particular values of bath temperature and light pulse energy the magnitude of the light effect, as measured by $\alpha T / d t$, decreases almost linearly with increasing $\lambda$ until a limiting value is reached beyond which the light induced cooling, if any, is below the sensitivity limits of our experiment.

\section{PHOTOGRAPHIC STUDIES}

In order to verify our hypothesis that the light effect was associated with the initiation of additional boiling at the solid-liquid helium interface, we initiated a series of photographic studies (Lezak 1983). The photographs were taken with a thirty-five millimeter single lens reflex camera fitted with a macro lens and extension tubes so as to form an approximately one-half life size image of the crystal which is inside the unsilvered walls of our Pyrex dewar system. We prevent water condensation on the outermost dewar surface by passing a steady stream of air past the dewar and we suppress normal boiling in both the helium inner and nitrogen outer dewars by momentarily slightly 


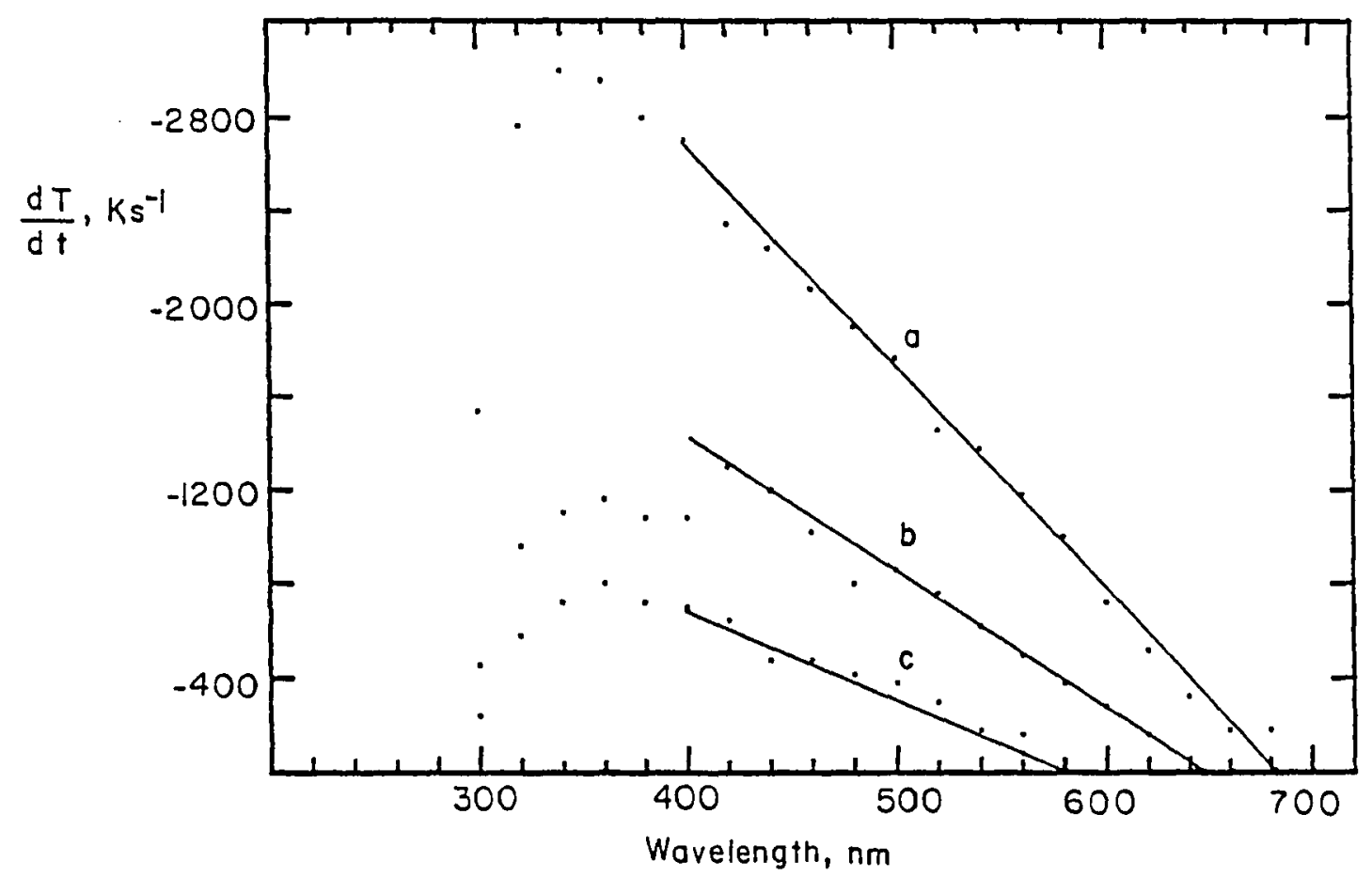

Figure 4: Light effect vs light wavelength. For wavelengths greater than $380 \mathrm{~nm}$ the light pulse energy is roughly constant. The three curves result from wavelength sweeps taken at different bath temperatures on the vapor pressure curve. The vertical scale is the average value of $\mathrm{dT} / \mathrm{dt}$ over 0.1 to $0.4 \mathrm{~ms}$ after the light flash. Curve a is at $2.6 \mathrm{~K}$ and has an intercept calculated from a 1 inear least squares fit of $684 \mathrm{~nm}$. Curve $b$ is at $3.0 \mathrm{~K}$ and has an intercept of $643 \mathrm{~nm}$, and curve $\bar{c}$ is at $3.4 \mathrm{~K}$ and has an intercept of $598 \mathrm{~nm}$. The tendency for $d T / d t$ to decrease at the short wavelength end is due to a combination of two factors: one, the light pulse energy of our source decreases rapidly below $380 \mathrm{~nm}$ and two, the transmission of the Pyrex dewar falls to almost zero between about $330 \mathrm{~nm}$ and $300 \mathrm{~nm}$. 
pressurizing at the time of each photograph.

\section{PHOTOGRAPHIC OBSERVATIONS}

Our photographic technique requires two separate light sources: one to initiate the light effect, and the second to photograph the crystal and its superheated liquid helium surface layer some time later. The photographic film is shielded from the first flash by passing the light through an interference type bandpass filter centered at $436 \mathrm{~nm}$ and by placing a long-pass gelatin filter, opaque to light of wavelength shorter than $520 \mathrm{~nm}$, in front of the camera lens. This combination allows enough light to reach the crystal to initiate a substantial light effect and yet ensures that the photographic image is entirely due to the second flash. In this experiment we used light sources with flash durations of approximately 5 us to ensure stop-action photography. The irradiance at the crystal is approximately $10 \mathrm{~mW} / \mathrm{cm}^{2}$. A translucent screen placed between the second light source and the crystal improves the quality of the back-lit image. We record the effect of the light flash by exposing the photographic film with a second flash triggered by a variable delay pulse generator which is synchronized with the first flash and the initiation of the light effect. We are thus able to record the visible effects of a light flash over a range of time delays from 200 to 2000 us after the flash. A typical photograph is shown in Fig. 5 a. This 

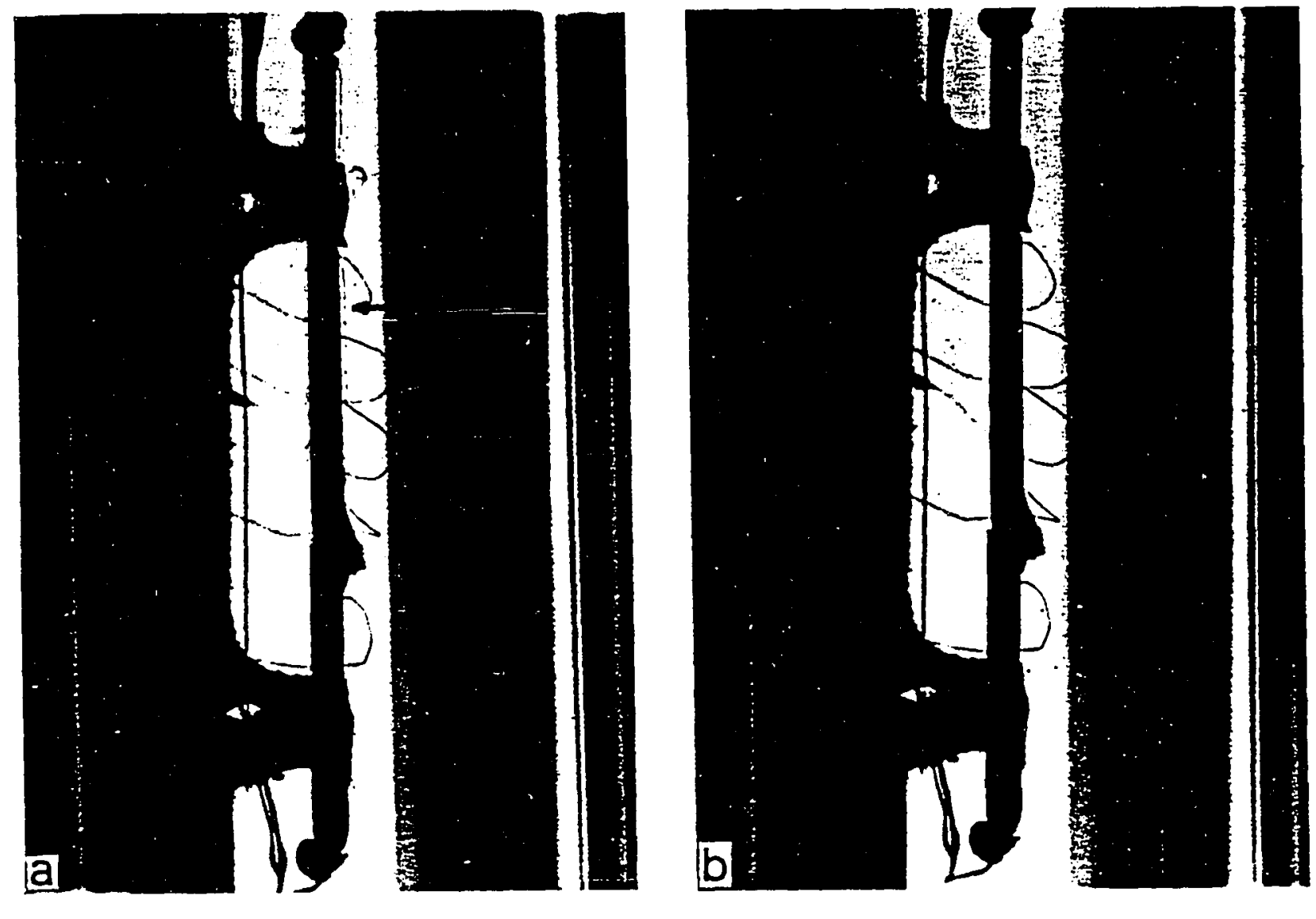

Figure 5: The bismuth crystal and crystal holder immersed in superheated liquid helium. Photograph a shows the system $1.0 \mathrm{~ms}$ after a light flash. Note the vapor film (indicated by the arrow) surrounding the crystal. In photograph b no light flash has been applied. Note the sharp edge of the crystal in contrast to photograph a. The dark lines coming off the surface of the crystal are potential leads for the four-terminal resistance measuring network. The crystal width is $2.1 \mathrm{~mm}$. 
photograph was taken $1.0 \mathrm{~ms}$ after the inital light flash and $4.0 \mathrm{~ms}$ after the start of the heating pulse. The bath temperature was $2.8 \mathrm{~K}$, the crystal temperature was approximately $4.0 \mathrm{~K}$ at the time of the initial flash and the heater power was $0.34 \mathrm{~W} / \mathrm{cm}^{2}$. The arrow on the figure indicates the border of a film of vapor surrounding the crystal with a thickness of 0.4 to $0.6 \mathrm{~mm}$. Figure $5 \underline{\mathrm{b}}$ is a photograph that was taken under similar circumstances except that the flash from the first light source had been eliminated. In this case there is no visible vapor layer. We conclude, therefore, that the vapor production is initiated by the first light flash and is associated with the cooling that we also observe to result from the light flash.

\section{PHOTOELECTRIC HYPOTHESIS}

Because our preliminary results suggested that there may be a long wavelength cutoff to the light effect we felt that one plausible mechanism for the production of bubble nuclei by the light could be a two step process in which, first, photoelectrons are detached from the bismuth by the action of the light and, second, the electron bubbles which are known to form in liquid helium serve as nuclei for ordinary vapor bubbles. (Sinha 1982a, Bennemann 1967) Since any phenomenon involving the plotoelectric effect would be expected to exhibit a long wavelength cut-off 
corresponding to the photoelectric work function of the presumed photocathode, an important test of the hypothesized photoelectron mechanism was to examine the wavelength dependence of the light effect characterized in Fig. 4 to see if such a cut-off exists and, if so, to compare it with the accepted value of the photoelectric threshold of the heater-thermometer material. Although approximate limiting values can be extrapolated from the graph, it is clear that no unique limiting value exists independent of the particular experimental conditions. We have also adapted a germanium crystal resistive element and a rhodium-iron wire to our transient thermometry technique. The light effect was again observed with these devices and, despite reduced sensitivity, the experimental results, including the absence of a clear and unique long wavelength cutoff, were consistent with those obtained with bismuth crystals as reported above.

\section{DISCUSSION OF PHOTOELECTRON HYPOTHESIS}

At first sight the wavelength dependence of the light effect as shown in Fig. 4 suggests a long wavelength cutoff. However, this apparent cut-off wavelength ranges from 400 to nearly $700 \mathrm{~nm}$ as the bath temperature is reduced or the light pulse energy is increased. Although it is possible that there is some single wavelength beyond which no light induced cooling could be observed, even in the most 
sensitive experiment, the existence of such a limiting value is clearly not a necessary conclusion based upon the results reported here. Additionally, the published values for the vacuum photoelectric threshold wavelength range around 294 $\mathrm{nm}$ for bismuth and $258 \mathrm{~nm}$ for germanium (Weast 1982). Although it might be expected that local variations of the work function of the surface would occur due to surface irregularities, impurities, reduced temperature, (Cardona 1978 ) or contact with liquid helium, (Woolf 1965, Northby 1967, Smith 1976) none of these factors appears capable of explaining the dramatic reduction in the photoelectric work function necessary to explain the long wavelength effect that we have found here. The evidence presented here leads us to effectively rule out the hypothesis that the vapor bubbles associated with the cooling observed in the light effect are nucleated by electrons which are ejected completely free of the solid surface through the photoelectric effect.

To further test the photoelectric hypothesis we placed a conducting screen approximately $1 \mathrm{~mm}$ away from one surface of a bismuth crystal heater-thermometer and found that the application of a $180 \mathrm{~V}$ potential between the screen and the crystal neither enhanced the light effect with the screen biased positive nor inhibited it with the screen biased negative. Furthermore, attempts to detect a photocurrent between the crystal and the positively biased screen with 
light of wavelengths ranging from 300 to $700 \mathrm{~nm}$, typical for our other experiments, were linsuccessful.

It is still. difficult to rule out a remaining related possibility. It is conceivable that an electron may be injected a short distance into the 1 iquid helium by the action of light of wavelength greater than the photoelectric cut-off wavelength. Such a quasi-free electron might then exist in the superheated liquid helium near the heater surface for a time long enough for an electron bubble to form before the electron was drawn back into the solid by the image force. It is unclear at this time what minimum photon energy would be necessary to produce such an electron and whether our electric field experiment, which gave negative results, was sensitive enough to be conclusive in this case.

\section{STEADY STATE EFFECTS}

We have observed that a flash of light applied to a system in quasi steady-state nucleate boiling results in an irreversible transition to a lower $\Delta^{\mathrm{T}}$ for any given heat flux, q (Lezak 1984b). Figure 6 shows a typical plot of the effect of slowly increasing heat flux to within approximateIy $80 \%$ of the peak nucleate boiling heat flux (PNBHF) and then slowly decreasing back to zero. It is clear that the value of $\Delta^{T}$ for any given $q$ is strongly dependent upon the thermal history of the heater-thermometer. This hysteresis 


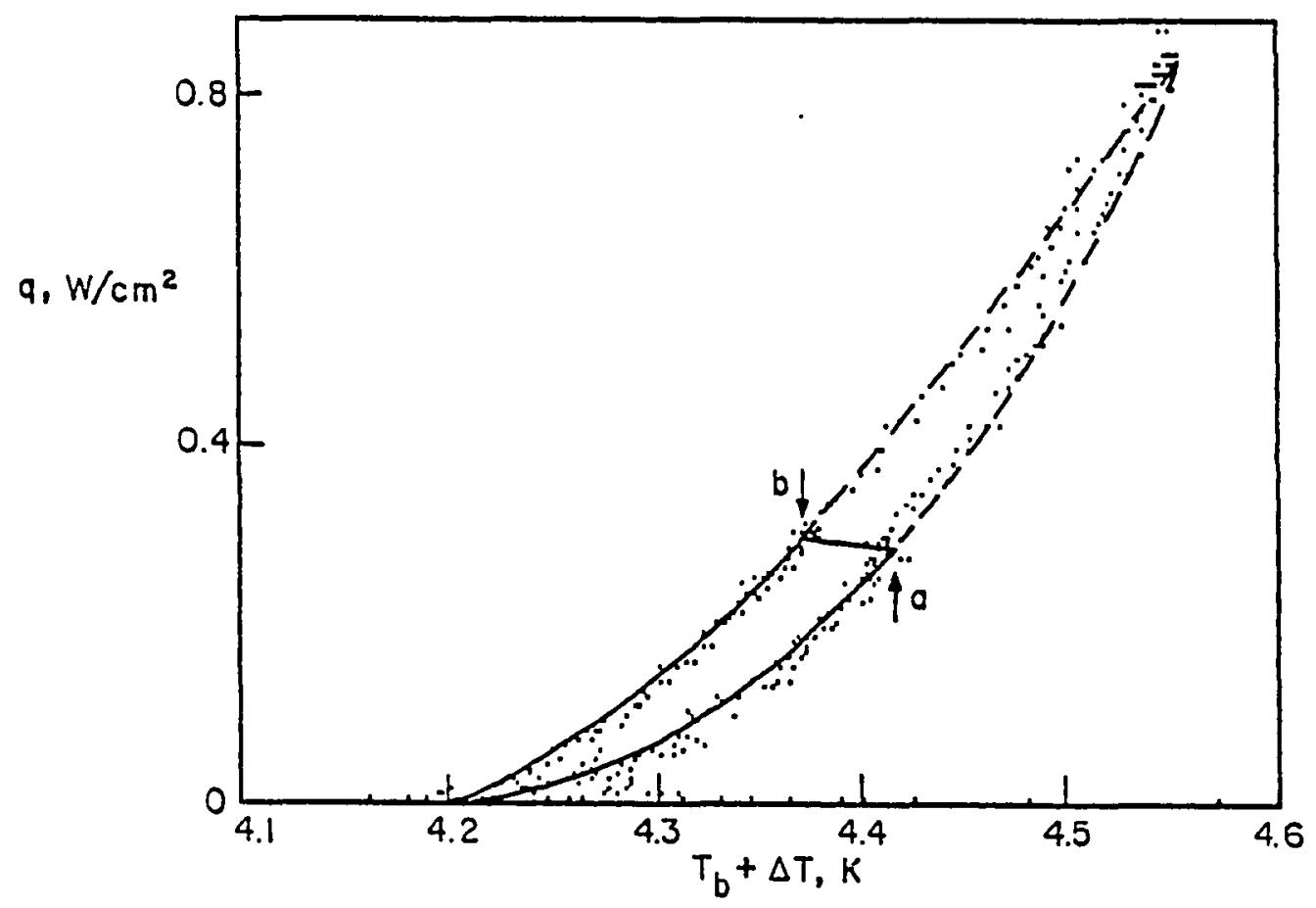

Figure 6: Quasi steady-state $q$ vs $\Delta^{T}$. A light flash applied at the point indicated by arrow a results in a rapid decrease in temperature for roughly constant heat flux to the point indicated by arrow $\underline{b}$. The slight increase in heat $f$ lux from points a to $\underline{b}$ is the result of the increase in crystal resistance as the temperature decreases. 
loop has a larger $\Delta^{T}$ for the increasing heat $f$ lux portion of the curve than for the decreasing portion.

The arrows $\underline{a}$ and $\underline{b}$ in Fig. 6 show the effect of applying a flash of light to the thermometer after the power has been slowly increased to approximately 408 of the PNBHF. The result is a reduction in superheat for the same heat flux to near that of the decreasing heat flux side of the hysteresis loop. A stronger light flash would result in a still greater reduction in superheat and a weaker flash would result in less. This effect, unlike the one observed with transient superheating, does not appear to show a temperature threshold. If the heat flux is high enough that the system is clearly in a nucleate boiling regime, then a flash of light will result in a reduction in total superheat to the hysteresis limits.

\section{APPLICATIONS}

Although the actual mechanism by which visible light influences nucleate boiling remains unclear the effect is nevertherless interesting and potentially useful. of immediate relevance in the study of heat transfer is the question of the effect of the light source in photographic studies of nucleate boiling. Our experiments point out the need to determine the extent, if any, to which the photographic process itself acts to induce nucleation in the system being studied. It is of course possible that the 
light effect occurs only in 1 iquid helium and therefore has little bearing on photographic studies of boiling in other liquids. There are reports, however, of light influencing the rate of condensation nucleation from vapor to liquid (Katz 1975) so this effect may be quite broad and is a subject that deserves further study.

It is also possible that this effect could be of significance both for practical cryogenic engineering and for the acquisition of fundamental knowledge of boiling heat transfer and nucleation in cryogenic liquids. For example, it might lead to methods of suppressing undesirable transient temperature excursions in the cryostatic stabilization of superconducting devices which are refrigerated in Iiquid helium.

We also feel that the light effect could be useful in studying the two-phase heat transfer that results from the sudden production of large numbers of vapor bubbles in a superheated liquid. Curves such as those shown if Fig. $1 \underline{b}$ and their calculated time derivatives describe the development of the additional heat flux following the application of a flash of light. This is a comparatively simple situation that could be used to test appropriate theoretical predictions.

Finally, there is the effect of 1 ight on the quasisteady state $q$ vs $\Delta^{T}$ curve. The hysteresis that is shown quite clearly in Fig. 6 has been observed as early as 1963. 
(Reeber 1963) Present theories suggest that the reduction in $\Delta^{T}$ for any given $q$ on the down side of the curve results from the preceding activation of additional nucleation sites. (Kosky 1968) It appears that a flash of light has the same effect as temporarily increasing the heater superheat and a careful study could yield information on the density and behavior of nucleation sites in steady state nucleate boiling. It has been reported that various surface treatments can reduce, and even reverse, this hysteresis and it would be interesting to study the effect of a flash of light on these surfaces (Butler 1970).

BUBBLE GROWTH DYNAMICS

As a further application of the light effect, we have used the photographic technique to study the development of the bubble layer associated with the light effect as a function of time after the light flash. This was done by evaluating photographs taken at different time delays after the initial light flash but in otherwise identical circumstances. Our results are summarized in Fig. 7 which is a $\log -\log$ plot of vapor film thickness vs time for three series of photographs taken at different bath temperatures. For times between 200 and 2000 us we may describe the vapor layer thickness, $\delta(t)$, by a power $\mathrm{law}$ of the form $\delta(t)=$ $C t^{n}$ where $n$ is the slope and $c$ is the antilog of the $y^{-}$ intercept of the $\log -\log$ graph. 


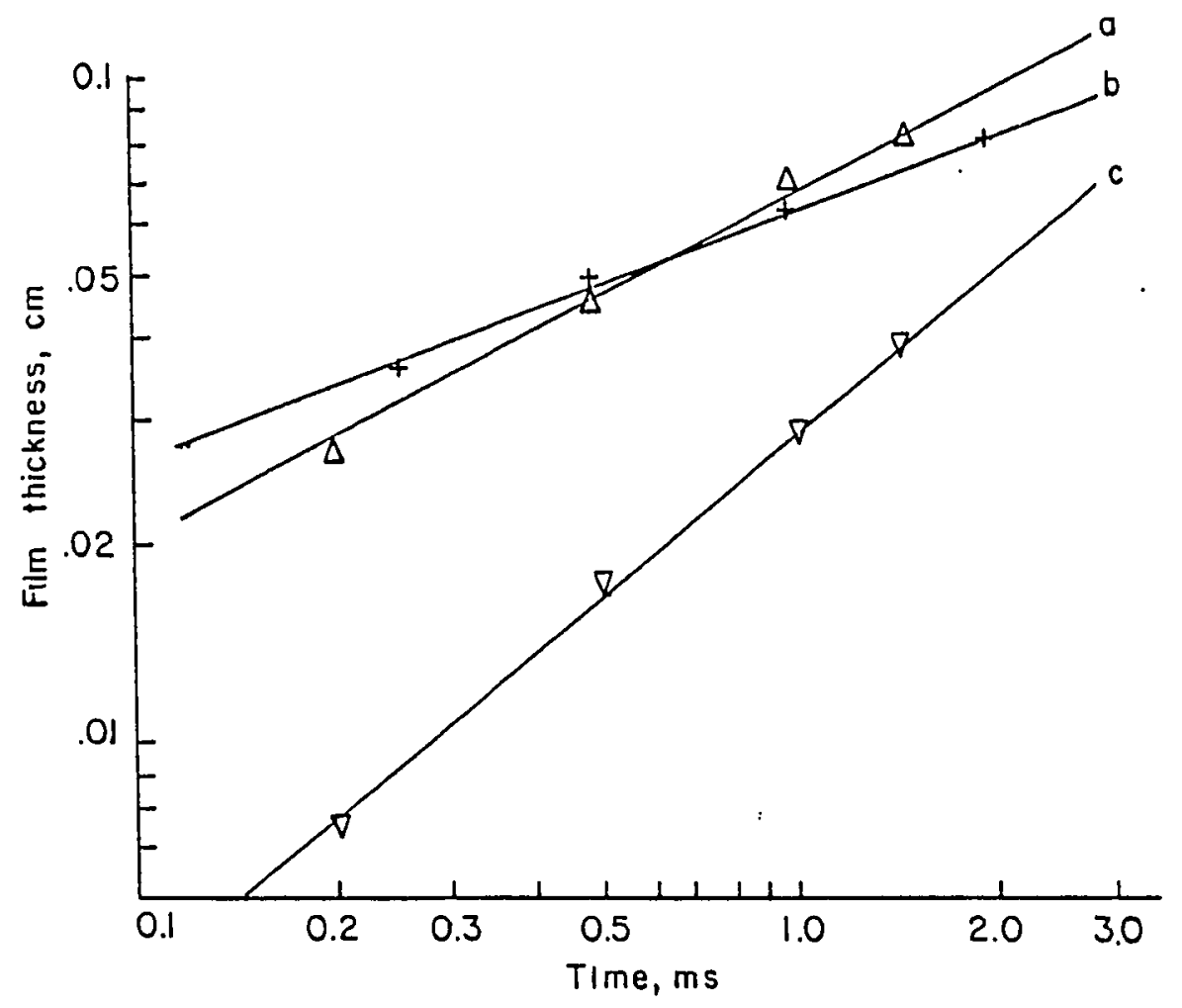

Figure 7: Log thickness vs log time after the intial light flash. Curve a was taken at a bath temperature of 2.2 $\mathrm{K}$. Curve $\underline{\mathrm{b}}$ was taken at a bath temperature of $2.4 \mathrm{~K}$ and curve $\underline{\underline{c}}$ was taken at a bath temperature of $2.8 \mathrm{~K}$. 
Although the environment of the bubbles in our study is very complicated, we expect that the bubble growth dynamics should be similar to those predicted for isobaric heat diffusion controlled growth of free spherical bubbles. Theoretical studies of this growth region have determined that the expected growth rate is $R(t)=2 C_{1}(x t)^{1 / 2}$ where the thermal diffusivity, $\alpha$, is given by the experimental conditions and the growth constant $c_{1}$ is an implicit function of the Jakob number $\left(p_{2} c_{2} / \rho_{1} h_{l v}\right)(\Delta T)$ and the liquid to vapor density ratio (A/pv). (Tong 1975, Mikic 1970, Plesset 1954, Birkhoff 1958) $\Delta^{T}$ is the liquid superheat, $C_{1}$ is the Iiquid heat capacity, and $h_{I v}$ is the heat of vaporization.

Previous experimental studies of pool boiling bubble growth in liquid helium have confirmed that the radius does increase as $t^{1 / 2}$ but do not give a consistent value for the growth constant $C_{1}($ Pavlov 1980$)$. Our own results for $n$ also tend to cluster around $n=1 / 2$. However, the growth constant $\mathrm{C}_{1}$, as estimated from our experimental data, varies from roughly 50 to 250. Because of the transient nature of our experiments we expect $c_{1}$ to vary with time but calculations based on theoretical work reported by Tong (1975) indicate that it should fall in the range 5-50. Our consistently high value of $\mathrm{c}_{1}$ could be taken as evidence that the interference between bubbles limits their full three dimensional spherical growth at the stage where they 
become visible in our experiment. 
CHAPTER III

TRANSIENT HEAT TRANSFER

INTRODUCTION

Studies of transient heat transfer in liquid helium have been and continue to be of great interest for both theoretical and practical applications. We have adapted our techniqes of transient magnetoresistive thermometry to examine two areas of Iiquid helium heat transfer of particular interest: the Kapitza limits to thermal boundary conduction and the delay to the onset of film boiling following the application of a large heat pulse. Although the primary applications for this work may be as a reference for other experiments such as our measurements of the homogeneous nucleation temperature, our studies of high temperature Kapitza thermal boundary resistance provide an important extension to our knowledge of a phenomenon which is otherwise difficult to measure. Studies of the time delay to the onset of film boiling are, on the other hand, of immediate practical importance in the design of systems-typically superconducting devices--which are cooled by liquid helium and subject to power transients. 


\section{MEASUREMENT OF THE KAPITZA THERMAL BOUNDARY CONDUCTANCE FROM BISMUTH TO NORMAL IIQUID HELIUM ISOTOPES 3 AND 4 AT THEIR HOMOGENEOUS NUCLEATION TEMPERATURES}

\section{INTRODUCTION}

Measurements of the thermal boundary conductance, $h_{k}$, between solids and liquid helium--Kapitza conductance--have traditionally been made at bath temperatures below the superfluid transition temperature of liquid helium isotope 4 (Snyder 1969). This is because a stable temperature reference, such as is provided by a large superfluid bath, greatly facilitates these measurements (Kim 1983). We have used the homogeneous nucleation temperature of liquid helium to provide another reference point. This has allowed

- measurements of $h_{k}$ in the temperature range of 4.0 to $4.6 \mathrm{~K}$ in helium-4 and 2.5 to $2.8 \mathrm{~K}$ in helium-3. Our results are roughly consistent with an extrapolation of previously reported temperature dependent results in the range $1-2 \mathrm{~K}$ (Cheeke 1973).

\section{THEORETICAL BACKGROUND}

In experiments involving transient superheating of a solid immersed in normal liquid helium, the observed maximum superheat becomes relatively constant at applied powers, $q$, 
greater than approximately $50 \mathrm{~mW} / \mathrm{cm}^{2}$ (Sinha 1982b). This is explained by the sudden onset of thermally activated homogeneous nucleation, and the maximum liquid superheat is the homogeneous nucleation temperature, $T_{h}$, of the liquid. This temperature is predicted by the so-called "classical" nucleation theories, such as those of Becker and Doring (Skripov 1974). Because the predicted nucleation rate varies by several orders of magnitude within a narrow range of superheating, this prediction effectively defines a kinetic limit of metastability which provides a surface layer of liquid helium at a temperature, $T_{h}$, that is substantially independent of heating rate (Sinha 1982b). Thus the heat flux dependent maximum solid superheat will be $\mathrm{T}_{\mathrm{h}}+\Delta^{\mathrm{T}} \mathrm{k}$ and the Kapitza conductance, $\mathrm{h}_{\mathrm{k}}$, may be calculated from the equation:

$$
\mathrm{h}_{\mathrm{k}}=\mathrm{q} / \Delta^{\mathrm{T}} \mathrm{k}
$$

\section{OBSERVATIONS}

Figure 8 is a graph of measured maximum superheat temperature as a function of calculated heat flux. Although there are several possible factors that could contribute to the observed increase in maximum superheat with increasing heater power, such as nucleation and bubble growth kinetics (Skripov 1974), and the finite response time and internal temperature gradients of our bismuth crystal thermometer (Brodie 1981, Sinha 1982b), preliminary calculations suggest 

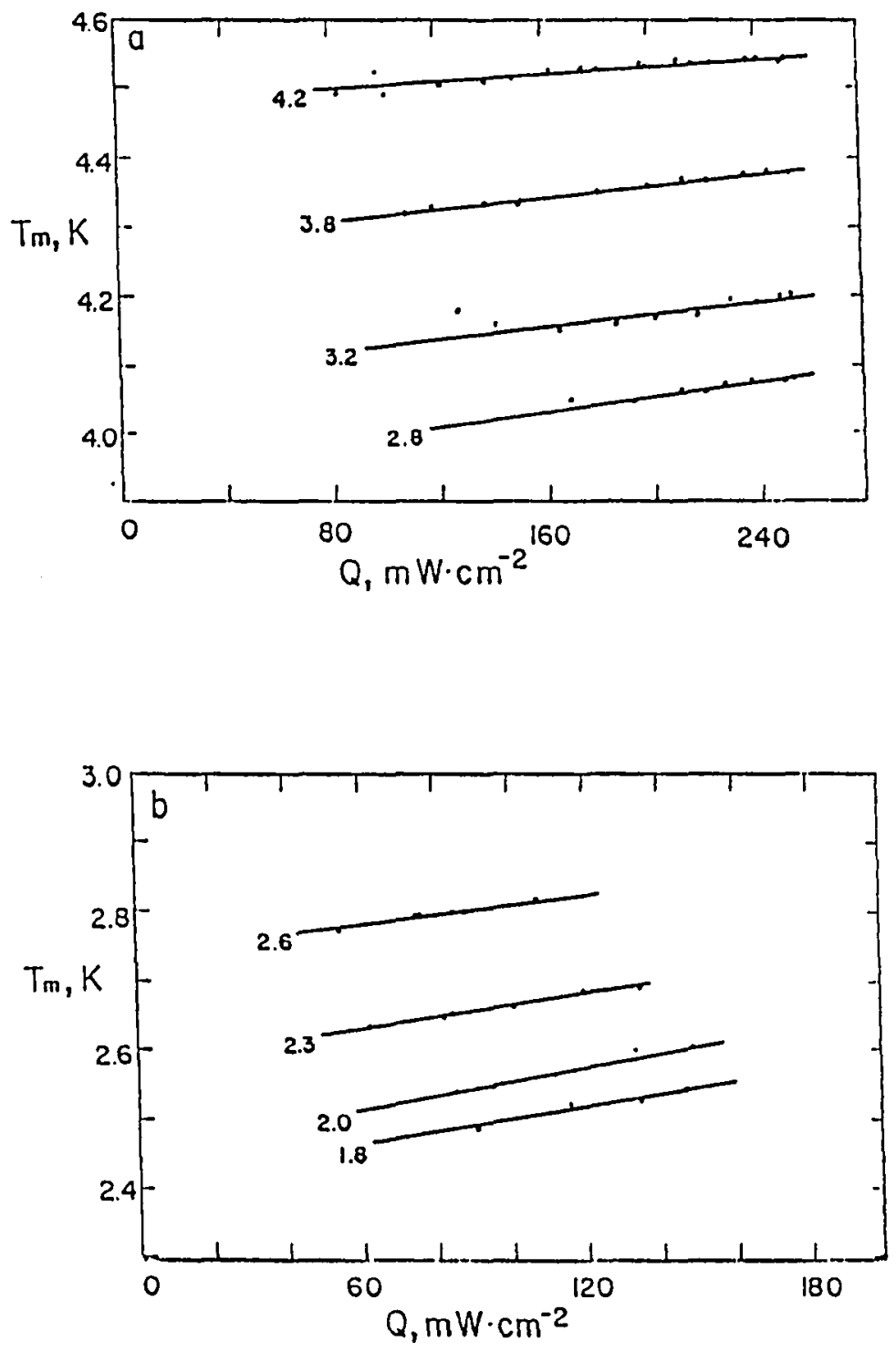

Figure 8: Measured maximum superheat vs heat flux at various bath temperatures. The data in Fig. 1a were taken in helium-4 and the data in Fig. $1 \underline{b}$ were taken in helium-3. 
that Kapitza conductance is the overwhelming factor. We have also measured the Kapitza conduction limits at the interface of our bismuth crystal heater-thermometer and superfluid 1 iquid helium-4 in the bath temperature range $\mathrm{T}_{\mathrm{b}}=$ 1.42 to $2.16 \mathrm{~K}$. Because there is effectively no superheating in the superfluid bath the temperature of the solid reaches a temperature $T_{b}+\Delta^{T}$ within a few tens of microseconds after the application of a step function heating current. Because the maximum powers used in this experiment typically resulted in $\Delta^{T}{ }_{k}^{\prime} s$ approximately equal to $\mathrm{T}_{\mathrm{b}}$, it was necessary to define an effective Kapitza conductance including the additional terms in the expansion $(\mathrm{T}+\Delta \mathrm{T})^{4}-\mathrm{T}^{4}$ (Snyder 1969) giving:

$$
h_{k}^{\prime}=h_{k}\left[1+(3 / 2)\left(\Delta^{T} / T\right)+(\Delta T / T)^{2}+(1 / 4)(\Delta T / T)^{3}\right]
$$

It appears that Eq. 5 actually overcorrects the data slightly giving a corrected Kapitza conductance that decreases somewhat as $\Delta^{\mathrm{T}}$ is increased. We have fit these data, along with our higher temperature results, to an empirical power law form in order to determine the dependence of Kapitza conductance with temperature. The best fit for all of our data between 1.4 and $4.5 \mathrm{~K}$ is obtained using the coefficients $h_{k}=0.18 * T^{1.8}$. This value has been reported elsewhere to be $h_{k}=0.16 * \mathrm{~T}^{2.4}$ in the temperature range $1-2 \mathrm{~K}$ (Cheeke 1973). In view of the experimental uncertainties, these measurements appear to be in rough agreement. It should be noted that our measure- 
ments were made in a magnetic field of $0.8 \mathrm{~T}$ which is necessary to provide the magneto-resistance of the bismuth crystal heater-thermometer. 
TRANSIENT SOLID-LIQUID HELIUM HEAT TRANSFER AND

THE TIME DELAY TO THE ONSET OF FILM BOILING

INTRODUCTION

Heat transfer from a superheated solid to liquid helium I takes place by a variety of mechanisms. For heat fluxes less than $0.6-1.0 \mathrm{~W} / \mathrm{cm}^{2}$ the maximum average temperature of the heater surface, $T_{\max }$ stays near or below the homogeneous nucleation temperature, $T_{h}$, of the liquid and is thus only moderately superheated (Sinha 1982d, Tong 1975). If a persistent heat flux beyond this range is maintained the liquid develops a film boiling configuration and the superheat can increase by several orders of magnitude. This phenomenon is called departure from nucleate boiling (DNB) and the critical heat $f l u x$ is called the peak nucleate boiling heat flux (PNBHF). Although roughly constant, this PNBHF shows some dependence on heater geometry and surface conditions as well as bath temperature and pressure. In typical applications in which liquid helium is used as a coolant for superconductors heat is occasionally generated by transient disturbances such as flux jumps or mechanical stresses. If the heat can not be dissipated so that the superconductor is stabilized and returned to its superconducting state before DNB occurs the disturbance can 
spread and result in potentially destructive burnout.

It has been previously reported that the PNBHF can be exceeded for short periods of time without the occurrence of DNB and the associated catastrophic superheat because latent heat is transported away from the surface through nucleate boiling in the short time, $t<t_{d}$, before a stable film boiling configuration develops. (Schmidt 1978, Metzger 1982, Steward 1978, Tsukamoto 1975, Giarratano 1983, Adler 1983, Sinha 1979) This paper reports the extension of those works over a wide range of bath temperatures. This has al lowed comparisons of our experimental results with a strongly temperature dependent theoretical prediction proposed by schmidt (1978) which related the delay times, $t_{d}$, to the applied heat flux, $Q_{\text {app }}(T)$ :

$$
Q_{\text {app }}(T)=2\left(\alpha / t_{\alpha}\right)^{1 / 2} \rho_{1} h_{1 v}
$$

where $\alpha(T)$ is the thermal diffusivity and $h_{l v}(T)$ is the latent heat of vaporization of liquid helium. The origin of this prediction is discussed as well as its physical meaning in terms of describing the conditions necessary for the development of a film boiling configuration.

EXPERIMENTAL TECHNIQUE

We have adapted a technique of bismuth magnetoresistive thermometry to this experiment. In this method we have exploited the temperature dependence in the magnetoresistance of bismuth to al low us to continuously monitor 
the temperature of a single crystal of bismuth which is heated by the application of a step function current pulse. Complete details of the bismuth thermometer and its associated circuitry can be found elsewhere (Brodie 1977, 1981). Because of its small heat capacity and large coefficients of thermal conductivity and diffusivity, bismuth is ideally suited to this type of transient thermometry. We estimate a temperature measurement uncertainty of less than $50 \mathrm{mK}$ and a thermal response time of well under 100 usec. The crystal has a cross section of roughly 2 by $2 \mathrm{~mm}$ and is approximately $30 \mathrm{~mm}$ in length.

\section{EXPERIMENTAL RESULTS}

Figure 9 is a plot of superheat vs time in a typical experiment. The temperature of the bismuth crystal heaterthermometer is seen to rise rapidly to $\mathrm{T}_{\max }$ where it remains roughly constant until the sudden rise in temperature marking DNB. We should note here that we have not corrected our results for Kapitza thermal boundary conductance limits so the heater-thermometer is actually measuring the bath temperature plus $\Delta \mathrm{T}_{\mathrm{k}}$. (Snyder 1969, Lezak in preparation) The magnetoresistance of bismuth decreases with increasing temperature so the heat $f l u x, Q$, which is approximately equal to the applied power in our constant current experiments, $\left(Q=I^{2} * R(T)\right)$ is self-limiting and prevents burnout and the associated damage that could result. 


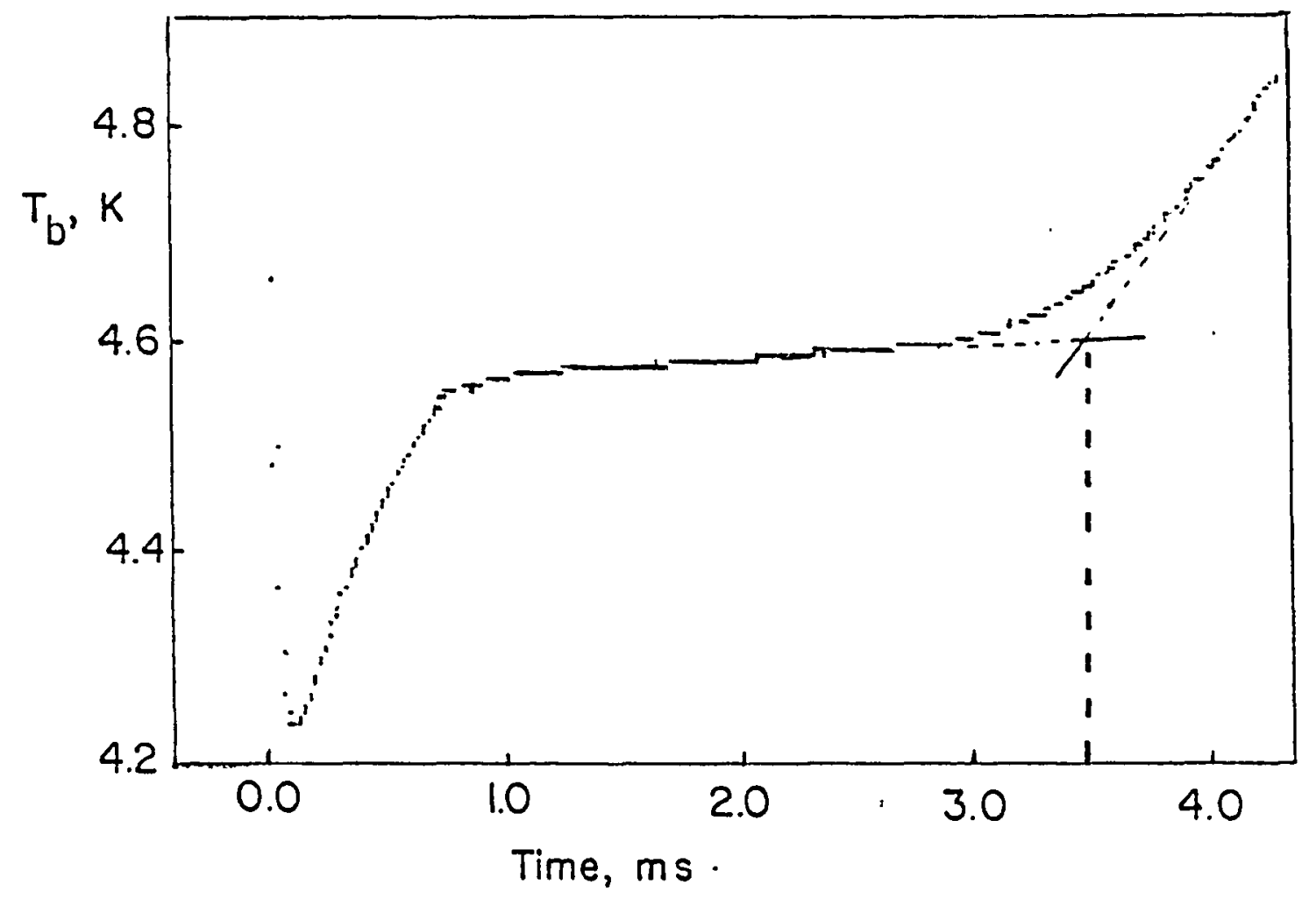

Figure 9: Superheat $\Delta^{T}$ vs time history for an applied heat flux greater than the peak nucleate boiling heat flux (PNBHF). The delay times, $t_{d}$, reported in the text are determined by visual inspection of these curves to determine an approximate midpoint between the nucleate boiling and film boiling regimes. 
Because we are interested in the total heat energy transferred into the liquid helium, our measurements of the time delay begin with the start of the current pulse and continue until approximately the middle of the change in slope marking the onset of film boiling and the sudden increase in superheat.

Figure 10 is a $l o g-l o g$ plot of delay time, $t_{d}$, vs heat flux at several different bath temperatures. Each of these plots is approximately linear for delay times less that 1 to $2 \mathrm{msec}$ but show a tendency to level out as the delay time is increased by reducing the applied heat flux. Ultimately, as the heat $f I u x$ is reduced below the steady-state PNBHF nucleate boiling heat transfer can be maintained indefinite1y. Our results at a bath temperature of $4.2 \mathrm{~K}$ are in agreement with previously published results in experiments using a variety of thermometric techniques, solid surfaces, and geometries. (Schmidt 1978, Metzger 1982, Steward 1978, Tsukamoto 1975, Giarratano 1983, Adler 1983).

\section{THEORETICAL}

It should in principle be possible to calculate the delay time prior to the onset of film boiling from a microscopic consideration of the hydrodynamics and thermodynamics at the heater surface and in the superheated liquid helium layer. In practice, this is a turbulent two-phase problem in which conduction, vaporization, and convection play 
DELAY TIME US HEHT FLUX.

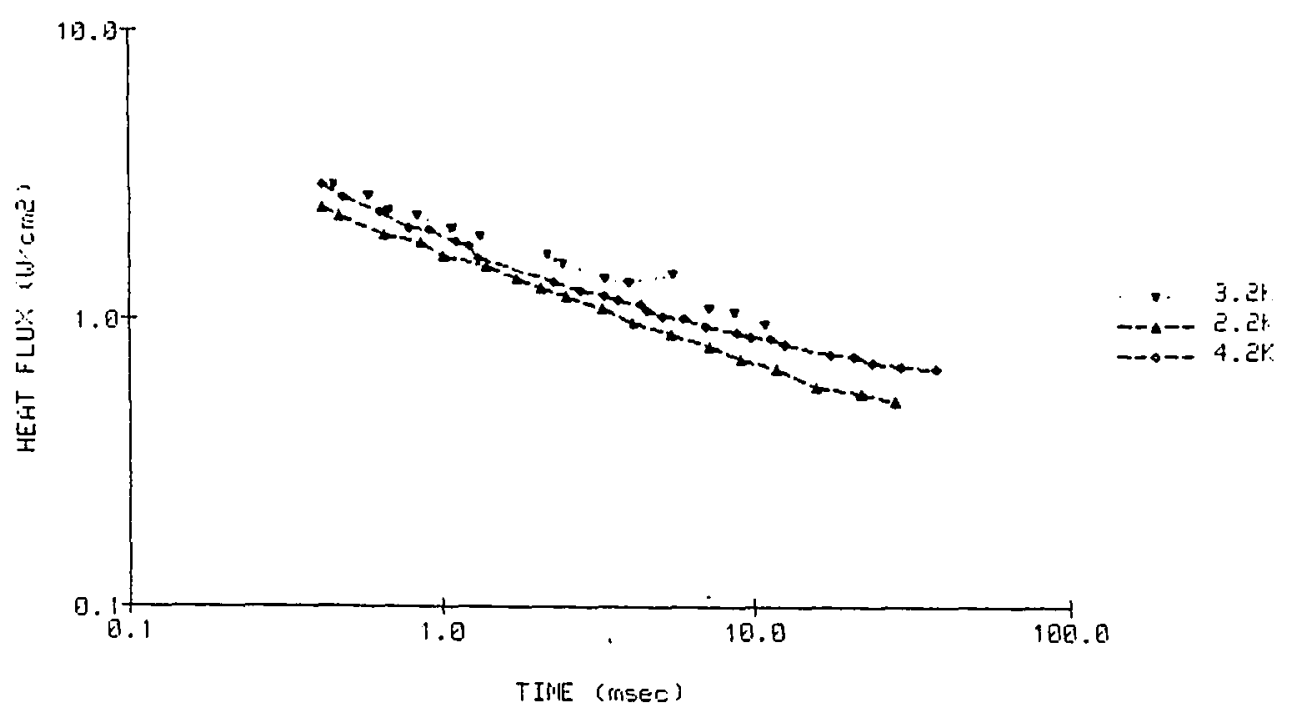

Figure 10: Log delay time vs log heat flux at several bath temperatures. These curves are approximately linear with slopes near $-1 / 2$ for delay times less that about one msec. The meaning of these slopes is discussed in the text. 
interrelated roles in the heat transfer process. We do not expect, therefore, to solve this problem exactly.

Calculations of the steady state values of the PNBHF have been made under a number of assumptions, many based on hydrodynamic wave motion theory, to establish a range of stable vapor layer thicknesses (Tong 1975). It remains unclear whether these approashes could be applied successfully to the transient case. One somewhat simplified approach to the problem of determining $t_{d}$ would be to determine the minimum quantity of energy necessary to produce a vapor layer meeting the hydrodynamic criteria for stability. Such a calculation would still have to account for energy lost by conduction, convection, and radiation as well as the nonequilibrium nature of the growing vapor layer.

An alternative approach neglects convective and radiative heat transfer and merely solves the simultaneous problem of determining the energy necessary to fully evaporate a superheated layer with a thickness $x(t)$ estimated by the heat diffusion equation:

$$
\begin{aligned}
& x(t)=2(\alpha t)^{1 / 2} . \\
& \int_{c}^{i d} Q(\tau) d \tau=Q_{a p p} t_{d}=x(t) \rho_{1} h_{1 v}
\end{aligned}
$$

so, having assumed $Q_{a p p}=$ constant, and solving for $Q$, we derive Eq. 6 :

$$
Q_{\text {app }}=2\left(\alpha / t_{d}\right)^{1 / 2} \rho_{1} h_{1 v} \text {. }
$$

This equation can be solved with numerical values at 
$4.2 \mathrm{~K}$ to obtain $\mathrm{Q}=0.1 \mathrm{Wsec}-1 / 2 \mathrm{~cm}^{-2}$ which is a factor of two above the usually reported values in the range of $t_{d}$ between .01 and $1.0 \mathrm{msec}$ (Touloukian 1979). Most important is the fact that the predicted exponent of the time, $-1 / 2$, is well supported by the experimental data. That such a crudely derived formula provides an accurate determination of the dependence of the delay time on applied power may at first seem surprising. It may well be, however, that for high heat fluxes and short delay times this equation may more accurately represent the limiting physical factors in the development of a film boiling configuration than the presumption of some minimum stable film thickness based upon quasi-steady state hydrodynamic calculations.

This can be seen from an examination of the development of the temperature and energy distribution in the superheated helium layer following the application of a step function heating pulse. From the time that the heaterthermometer reaches the homogeneous nucleation temperature of the Iiquid to the point that DNB occurs the superheat is roughly constant so the total heat flux, $Q_{t}=Q_{V}+Q_{C}$, where $Q_{V}$ is the heat $f I u x$ due to conduction and $Q_{C}$ is that due to vaporization near the surface, is also constant and equal to the applied power. Because the rate of thermally activated nucleation is highly sensitive to temperature variations around the homogeneous nucleation temperature, we find that vapor is produced at exactly the rate necessary to maintain 
that temperature through a feedback mechanism that is highly insensitive to the value of $Q_{t}$. The slight upward slope to the $\Delta^{T}$ vs time curve is probably due to the increasing fraction of vapor on the surface of the heater-thermometer, which measures the average surface temperature, and the local 'hot spots' which these voids produce. Eventually, enough vapor is formed in the thin superheated layer adjacent to the heater-thermometer surface to effectively inhibit conductive heat transfer into the bulk liquid and prevent the transfer of latent heat through vaporization. At this time, the temperature of the heater-thermometer must suddenly increase to maintain a balance between the applied power and the actual heat $f l u x$ and DNB is observed to occur. If we assume that the energy density in the superheated layer is roughly independent of distance from the surface of the heater because of turbulence and microconvection then the time at which DNB occurs will be exactly that time predicted by Eq. 6. It is well known, however, that DNB can occur with only partial vapor coverage of the heated surface (Tong 1975). Our experimental results suggest that this occurs when approximately 508 of the heated surface is coated with vapor. Our empirically corrected Eq. 6 thus becomes :

$$
Q_{\text {app }}=\left(\alpha / t_{d}\right)^{1 / 2} \rho_{1} h_{1 v^{\prime}}
$$

which not on Iy predicts the correct slope of the Q vs td curve but the correct intercept as well. 
The corrected theory can be summarized as follows: DNB will occur following the application of a step-function heating current after enough heat has flowed into the helium to vaporize $1 / 2$ the liquid in a layer 2 (ut) $1 / 2$ thick. This theory loses applicability for times greater than 1 to 2 msec, after which thermally and dynamically driven convection from bubble departure draw energy out of the superheated layer. We would also expect this equation to lose validity for times much less that $.01 \mathrm{msec}$ in which case the inital formation of vapor at the heater surface would be so catastrophic and pervasive that the hydrodynamics of the advancing vapor front would play a substantial role and film boiling would be immediate.

\section{DISCUSSION OF RESULTS}

One outstanding feature of Eq. 9 is that there is an expected dependence on two rather strongly temperature dependent quantities; $\alpha$, and $\rho_{1} h_{1 V^{*}}$ In order to test this dependence we have taken data over the range of bath temperatures extending from just above the $\lambda$-transition at $2.18 \mathrm{~K}$ to $4.2 \mathrm{~K}$. This is shown in Fig. 11 which includes much of the same data as Fig. 10 but the data have been replotted to demonstrate the effect on delay time of varying bath temperature at various constant heat fluxes. The open squares on Fig. 12 represents a plot of Eq. 9 for a heat flux of 1.4 $\mathrm{W} / \mathrm{cm}^{2}$. Although the fit to our the experimental results 
EATH TEMPERATURE US DELAY TIME

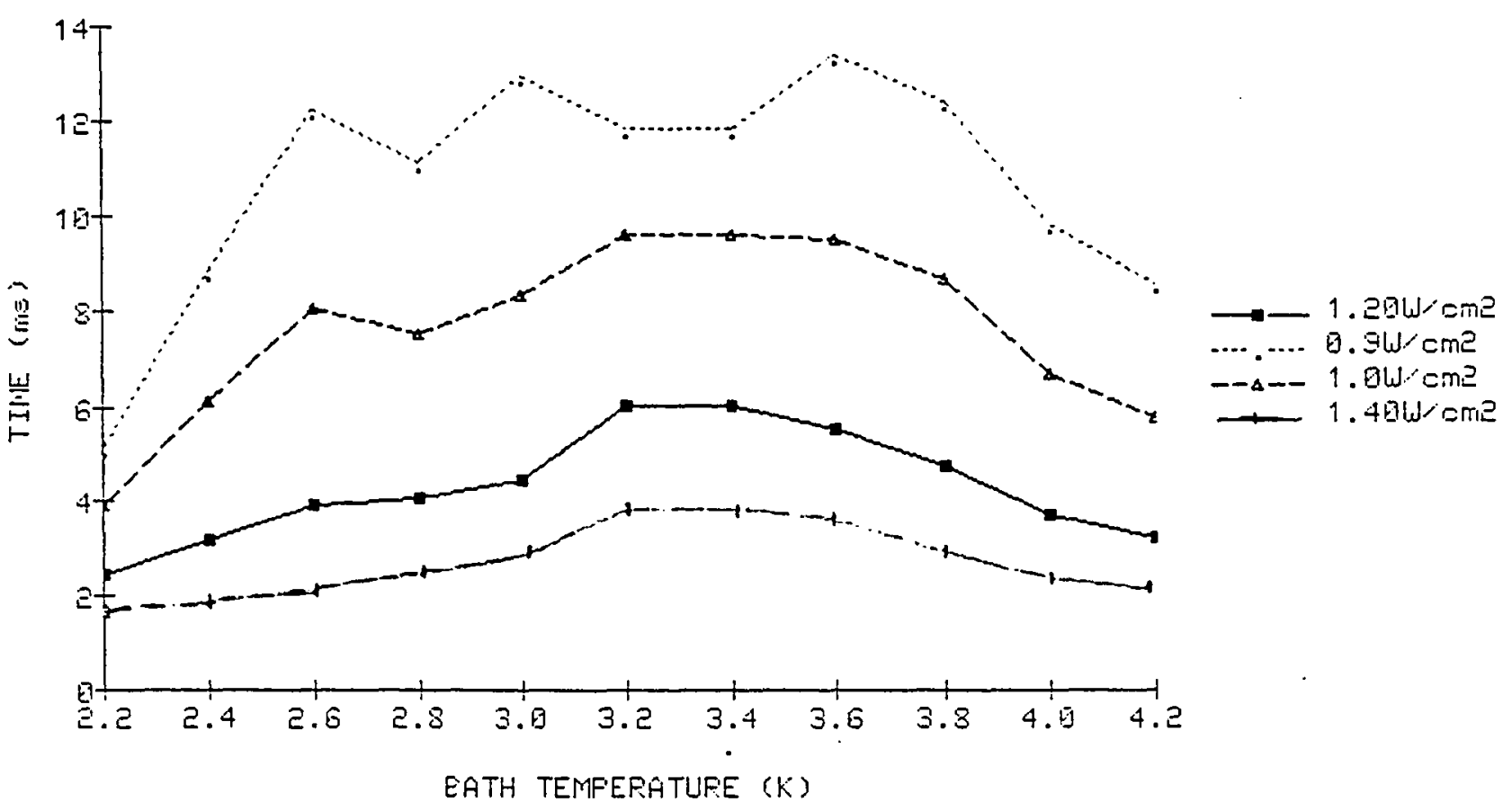

Figure 11: Delay time vs bath temperature for various heat fluxes. The data points have been generated from least squares polynomial fits to data in the form shown in Fig. 10 . 


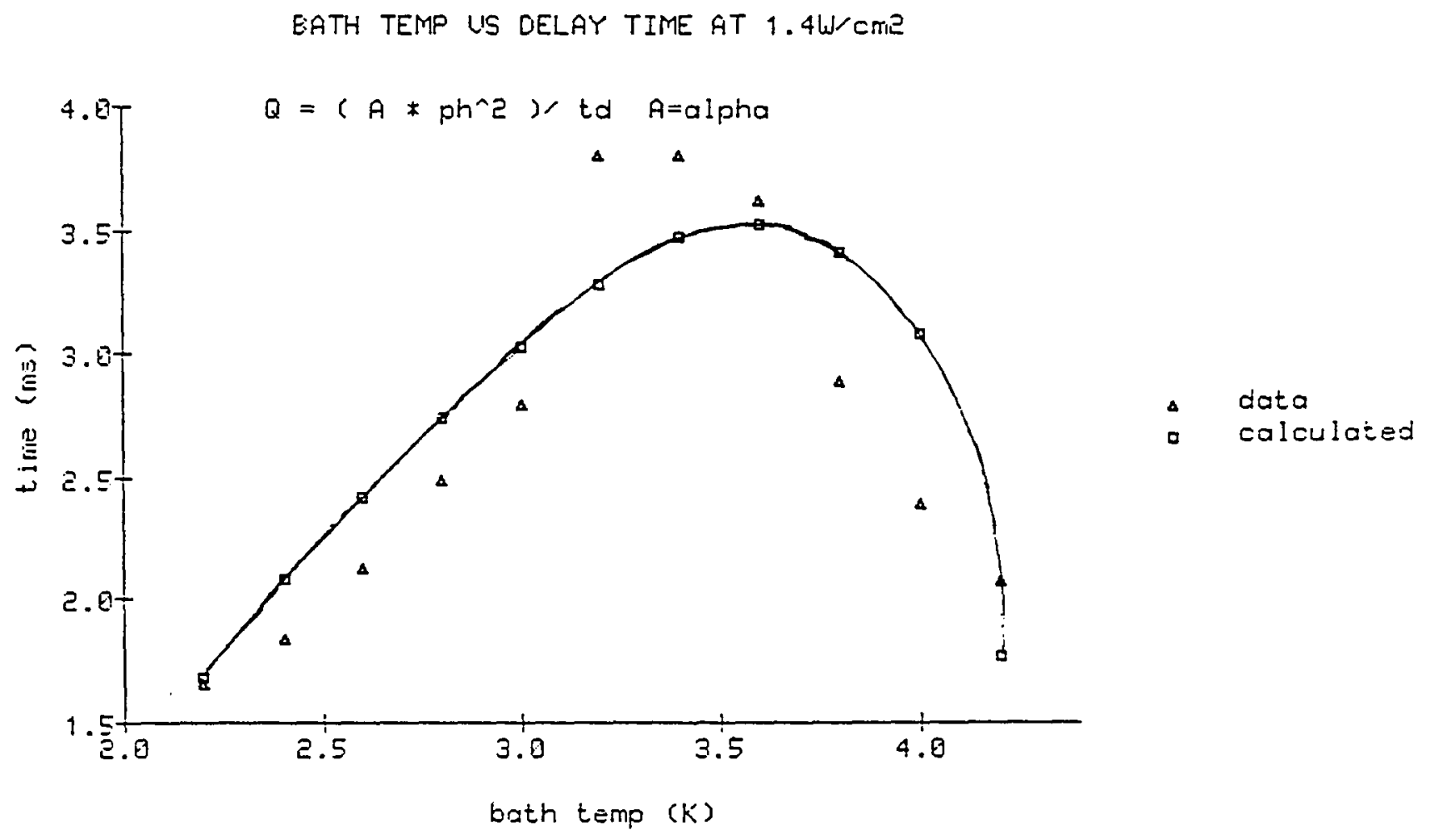

Figure 12: Bath temperature vs delay time at $1.4 \mathrm{~W} / \mathrm{cm}^{2}$. The open squares represent a plot of Eq. 9 for the same heat flux. 
for that heat $f l u x$, also included in the figure, is not precise the similarity is quite dramatic.

\section{CONCLUSION}

We conclude that for times less that $1 \mathrm{msec}, \mathrm{Eq} .9$ both provides an accurate predictor for the delay time prior to the onset of film boiling resulting from the application of a step-function heat pulse and accurately represents those physical factors determining that time. This suggests that the crucial factor in determining the time delay to the onset of film boiling is the competition between sensible heat transfer into the bulk liquid by diffusion and the vapor that is formed in the liquid as a result. For times longer than 1 to $2 \mathrm{msec}$ the data suggest that increasing amounts of energy are necessary to produce a film boiling configuration with the limiting case of no film boiling for applied heat fluxes lower than the steady-state PNBHF. This is due to the increasing importance of macroscopic convection and turbulence in moving heat out of the diffusion limited layer as time progresses. The results in this range still suggest that there is a strong dependence on the factor $(\alpha(T))^{n * \rho_{l}(T) h_{l V}(T)}$ but the exponent in time is substantially smaller that $1 / 2$. It is conceiveable that a further analysis, including a term for convection in the heat transfer equation, could provide a better fit to this large $t_{\mathrm{d}}$ data. 
CHAPTER IV

HOMOGENEOUS NUCLEATION TEMPERATURE OF ${ }^{3}$ He

\section{INTRODUCTION}

The classical theory of kinetics involved in the nucleation of one phase from another has received much attention since its original formulation by volmer and weber (1926), Becker and Doring (1935), and others (Blander and Katz 1975). Such phase transitions are associated with the existence of a thermodynamic barrier to the formation of droplets of a second phase in a homogeneous metastable parent phase. One application of this theory is in the prediction of an effective kinetic limit of metastability of superheated liquids. Successful measurements of this limit, called the homogeneous nucleation temperature, have recently been extended from the warmer cryogens into hydrogen and helium-4 (Sinha 1982b, Nishigaki 1983, Hord 1964). This verification was of considerable interest because hydrogen and helium-4 differ from the warmer cryogens in that they are effectively quantum liquids whose de Broglie wavelength, $\mathrm{h} /(\mathrm{me})^{1 / 2}$, is sizeable in comparison to the interatomic distance. The relative quantum mechanical nature cf these liquids can be simply scaled by the magnitude of the de Boer 
parameter $(\Lambda)\left[\Lambda=h / \sigma(m \epsilon)^{1 / 2}\right.$, where $h$ is Planck's constant, $m$ is the atomic mass, and $\epsilon$ and $\sigma$ are the Lennard-Jones parameters representing, respectively, the strength and range of the interatomic potential]. To put this scale into perspective, the so-called classical cryogens; argon, krypton, and xenon all have de Boer parameters, 1 , less than 0.2 while the values of $\wedge$ for hydrogen and helium- 4 are 1.7 and 2.7 , respectively. This verification of classical nucleation theory for superheating experiments in quantum liquids represented a powerful and not altogether expected success for that theory. This is in contrast to the case of tensile strength experiments where the predicted ultimate limits have never been observed for the cryogens (Beams 1956, 1959, Marston 1976).

Although the values of the homogeneous nucleation temperature have been correctly calculated by classical nucleation theory for these quantum liquids, they have been shown to differ substantially from predictions based on the approximate universal relation of the corresponding states approach:

$$
(1-\mathrm{Th} / \mathrm{TC})=0.14 *(1-\mathrm{P} / \mathrm{PC})^{0.92} \text {, }
$$

where $T_{h}$ is the homogeneous nucleation temperature, $T_{C}$ is the critical temperature, $\mathrm{P}$ is the saturation bath pressure and $P_{C}$ is the critical pressure (Sinha $1982 \mathrm{~b}$, Nishigaki 1983). This is shown in Fig. 13 which is a plot of the reduced homogeneous nucleation temperature, $\mathrm{T}_{\mathrm{h}} / \mathrm{T}_{\mathrm{C}^{\prime}}$ vs 


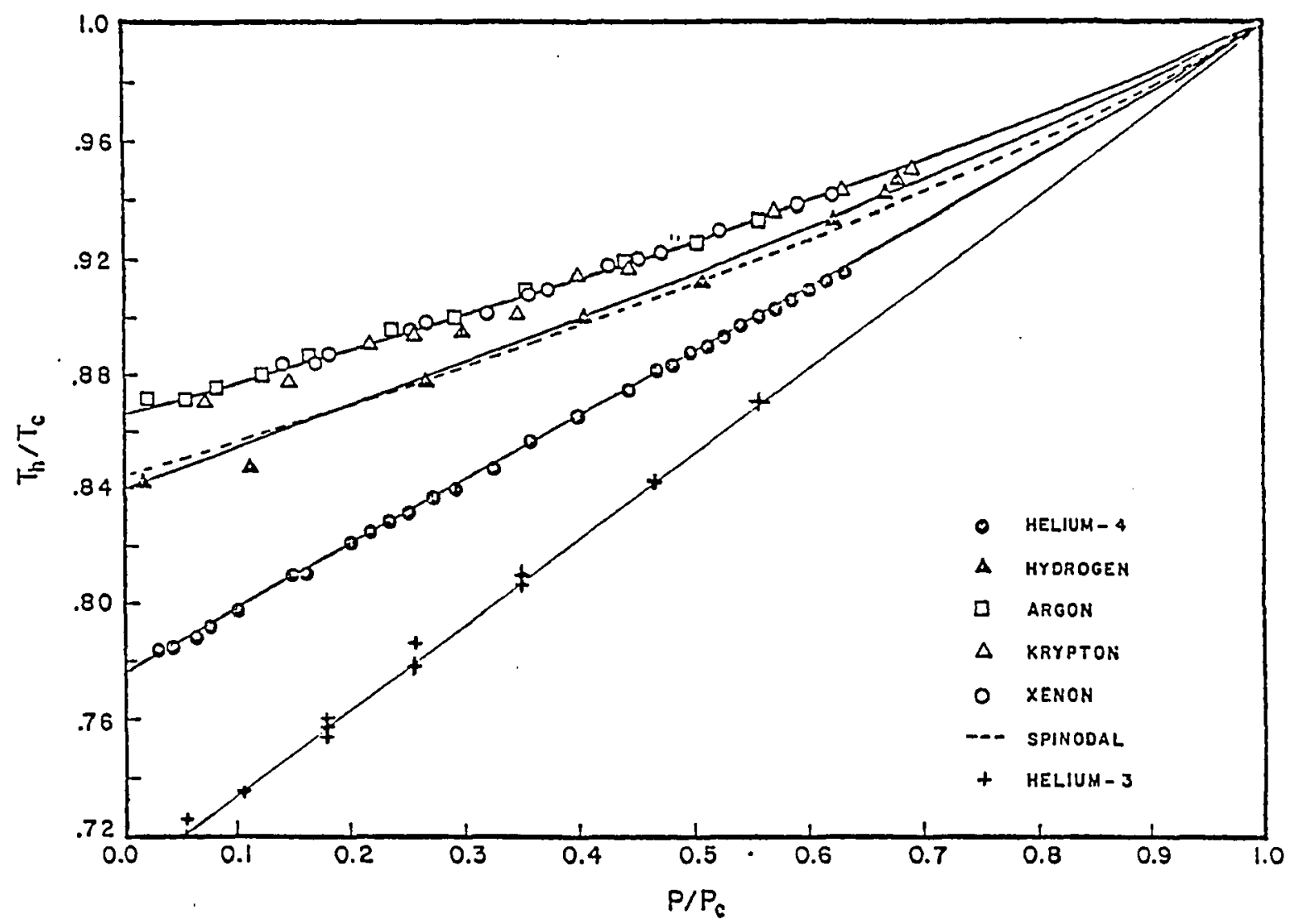

Figure 13: Scaled homogeneous nucleation temperature $\mathrm{T}_{\mathrm{h}} / \mathrm{T}_{\mathrm{C}}$ vs scaled pressure $P / P_{C}$. The homogeneous nucleation temperature data are presented as a function of the bath pressure for $\mathrm{Ar}, \mathrm{Kr}, \mathrm{Xe}, \mathrm{H}_{2}$, and ${ }^{4} \mathrm{He}$. The solid 1 ines represent the theoretical predictions of Th from Eq. 3 for eąch liquid. With the exception of the data points for ${ }^{3} \mathrm{He}$ which represent our own work, this curve is taken from Fig. 4 of Sinha, $1982 \mathrm{c}$. 
reduced bath pressure, $: P / P_{C}$, for several classical and quantum liquids. This deviation does not represent a breakdown in the classical corresponding states theory so much as a reflection of the relatively larger quantum mechanical influence on the thermophysical properties of the quantum liquids. Indeed, the magnitude of these deviations has been simply scaled to the de Boer parameter in a quantum extension to this theory of corresponding states (Sinha 1982b). One of the successes of this approach was the prediction of the homogeneous nucleation temperatures and other properties (Sinha 1982c) of Iiquids whose thermophsycial properties have not been well characterized by direct experimental observation, such as deuterium-tritium (DT) and tritium-tritium $\left(\mathrm{T}_{2}\right)$.

Following the verification of classical nucleation theory in helium-4, the next logical research step was to extend our studies of bubble nucleation in cryogenic liquids to the extreme limit of relative quantum influence and to still lower temperatures by measuring the homogeneous nucleation temperature of liquid helium-3. From a theoretical point of view, liquid helium-3 is interesting because it is the most strongly quantum liquid known, as measured by its de Boer parameter, and thus shows the largest deviation from the corresponding states predictions based on the classical liquids. Experimentally, it afforded the easiest access of any liquid to the lowest temperature. 
Also, it exhibits no superfluid transition down to very low temperature, an important practical consideration for our transient superheating method. The absence of superfluidity is important for another reason. This superheating work was only the first phase of a more comprehensive program to map out the entire boundary of the metastable region of helium three, including the negative pressure region where the superheating method must be at least supplemented by the tensile stress technique. Since the presence of bubbles originating at quantized vortex Iines has been suggested as one reason for the premature nucleation encountered in tensile stress experiments in liquid helium-4 (Finch 1966, McConnel1 1970), it seems prudent to move on to liquid helium-3 for further such studies, thus eliminating at least one possible reason for the failure to reach the predicted ultimate tensile strength.

\section{BACKGROUND}

Although attempts at experimental verification of Becker-Doring classical nucleation theory have met with considerable success, there are still some areas in which agreement between theory and experiment has not been observed.

For the case of nucleation of the vapor phase from the liquid the verification experiments have generally taken one of two forms. Many workers have used some type of super- 
heating technique in which the temperature of the liquid is raised to the homogeneous nucleation temperature, $T_{h}$, at constant pressure. Others have employed a variety of methods in which the pressure was lowered at constant temperature until nucleation of the vapor phase is observed. If the pressure can be reduced to a negative value before nucleation occurs, the tensile strength of the liquid may be measured. As a result of these efforts, it was definitively established that, for organic liquids, both superheating and tensile strength experiments yielded values of $T_{h}$ which were in good agreement with the predictions of the classical nucleation theory (Skripov 1979, Apfel 1971). However, for cryogenic liquids the situation is not so clear. For example, a variety of methods have been employed in attempts to measure the ultimate tensile strength of liquid argon, nitrogen, oxygen, and helium (Beams 1956, 1959, Marston 1976, Apfel 1981), but no values even approaching the predictions of classical nucleation theory have ever been observed. This discrepency has been attributed to a number of possible factors including impurity nuclei in the liquid or at the container walls, the production of ions by cosmic rays or radioactivity, and, in the case of liquid helium, the quantum nature of the liquid and the role of quantized vortex lines (Finch 1966, McConnell 1970). On the other hand, Skripov and co-workers have used superheating, expansion chamber, and acoustic 
cavitation techniques to stress liquid argon, krypton, and xenon, and have found good agreement with theory (Skripov 1979, Kaverin 1980, Baidakov 1981). However, it is important to note that both Skripov's work and our studies were restricted to the regime of positive pressure. Thus, at least in the warmer cryogens, the theoretical absolute limits have been acheived in experiments employing a variety of techniques at positive pressure, but no predicted ultimate tensile strengths requiring the application of tensile stress have yet been observed.

\section{THEORETICAL BACKGROUND}

In the classical nucleation theories, the formation of critical-sized nuclei in the metastable liquid as a result of thermal heterophase fluctuations is described in terms of a thermally activated transition across a thermodynamic energy barrier. The height of this energy barrier is determined by the minimum work of formation $\mathrm{w}_{\mathrm{cr}}$ of a critical (vapor filled) nucleus. The rate of formation of these critical nuclei is generally described by an Arrhenius-type expression:

$$
J=J_{0} \exp \left(-W_{C r} / k T\right),
$$

where $J_{0}$ is an attempt frequency determined by the dynamics of nucleus formation process, $T$ is the temperature, and $\mathrm{k}$ is the Boltzmann constant. This equation can be expressed more completely in a form recently given by Blander and Katz 
(1975):

$$
J=N\left(\frac{2 \sigma}{\pi m B}\right)^{\frac{1}{2}} \exp \left(\frac{-16 \pi \sigma^{3}}{3 k T\left(P_{e}-P_{1}\right)^{2} \delta^{2}}\right)
$$

where $J$ is the rate of formation of critical nuclei per unit volume per unit time, $\sigma$ is the bulk surface tension, $\mathrm{N}$ is the molecular density of the liquid, $m$ is the molecular mass, $B$ is a constant $(B=2 / 3$ in our superheating experiment), $\mathrm{P}_{e}$ is the equilibrium vapor pressure, and $\mathrm{P}_{1}$ is the ambient pressure. The Poynting correction factor $\delta$ relates $P_{V}$, the vapor pressure of the liquid under an ambient pressure $P_{1}$, to the equlibrium pressure: $\delta=\left(P_{v}-P_{1}\right) /\left(P_{e}-P_{1}\right)$. This equation is extremely sensitive to small changes in temperature so an effective homogeneous nucleation temperature can be defined by arbitrarily setting $\mathrm{J}=1$ critical sized nucleus per unit volume per second and then solving the resulting simplified equation for $P_{I}$ as a function of $T$. The kinetic coefficient in the preexponential factor of Eq. 3 does not include the modifications due to viscous or inertial forces that affect the dynamics of nuclei growth. However, these effects have been estimated to be negligible for calculations of the homogeneous nucleation temperature of helium-3 or helium-4 (Sinha 1980, 1982b). Additionally, the estimated relaxation time to establish steady state nucleation is several orders of magnitude smaller than the time scales considered here. From a theoretical standpoint, liquid helium-3 was 
especially interesting to study because it is the most strongly quantum liquid, as measured by its de Boer parameter $(\Lambda)$. The expected behavior of liquid helium-3 can be illustrated by referring again to Fig.13. The lines on the graph represent the theoretical predictions based on Eq. 3 while experimental data are represented by the various symbols. First, it will be noted that the values of $T_{h} / T_{C}$ for liquid helium isotopes 3 and 4 fall substantially below the data for the classical 1 iquids, argon, krypton, and xenon. It is important to emphasize that this separation of $\mathrm{T}_{\mathrm{h}} / \mathrm{T}_{\mathrm{C}}$ for Iiquid helium isotopes 3 and 4 from the classical law of corresponding states does not represent a breakdown of the theory. Rather, it results from the fact that the thermophysical properties involved in Eq. 12 are dependent upon the de Boer parameter (Bewilogua 1968, Sinha 1982C) which is significantly larger for helium than for the classical liquids, $\Lambda=2.68$ and 3.08 for helium-4 and helium3, respectively. For classical liquids, $\wedge$ is about 0.1 . Thus the large de Boer parameter of the liquid doesn't invalidate the theory but does serve to distinguish the homogeneous nucleation temperature of liquid helium-3 and the other quantum Iiquids from those of the classical liquids.

Lifshitz and Kagan (1972) were the first to point out that at temperatures close to absolute zero the appearance and the inital stage of growth of real nuclei are, from 
purely energetic considerations, impossible. Although such a mechanism has not been observed, they suggested that below a certain threshold temperature, $T^{*}$, the probability that a subcritical sized nucleus could tunnel through the classical energy barrier to nucleation is equal to that of a thermally activated transition over it. This probability increases with decreasing temperature as the energy, and thus the effective momentum of a quasiparticle representing the surface of a critical nucleus for liquid-to-vapor nucleation, decreases. If we consider that the quasiparticle has an effective de Broglie wavelength inversely proportional to its momentum we can see that tunneling becomes probable as the wavelength becomes greater than the classical width of the barrier. This has been predicted to occur at $0.2 \mathrm{~K}$ and at a pressure of negative 5.2 atmospheres in helium-3 and at $0.3 \mathrm{~K}$ and a pressure of negative 14.6 atmospheres for helium-4 (Sinha 1982b, Akulichev 1975). As will be seen later, our failure to observe premature nucleation in the temperature range of our work is consistent with these predictions. We conclude that a conventional thermally driven nucleation theory should be adequate in describing positive pressure liquid-to-vapor homogeneous nucleation in the helium isotope liquids without invoking any new nucleation mechanisms. None-the-less, these 'classical' positive pressure superheating experiments may be viewed as first steps towards an experimental test of the quantum tunneling 
nucleation theory.

\section{EXPERIMENTAI}

Our measurements were made using the technique of transient superheating pioneered by Brodie (Brodie 1977, 1978, 1981) and used by Sinha (1982b). However our experiments differ in some important ways from this technique. In particular, the prohibitive cost of helium-3 has forced us to recycle the vapor and necessitated the use of a smaller sized bismuth crystal in a more restrictive geometry than the Sinha group. This was due to the double constraints of fitting the experimental apparatus inside an already existing helium-4 cryomagnetic dewar and the very limited supply of helium-3 that was avaliable for 1 iquefaction. The experimental apparatus is shown schematically in Fig. 19. In addition, we believe that these constraints were also responsible for our inability to extend our measurements beyond a reduced pressure $\left(P / P_{C}\right)$ of about 0.5 because the restricted geometry reduced normal circulation. At reduced pressures greater than about 0.5 , we would observe that the coefficient of heat transfer, even for relatively low heat fluxes, was substantially reduced from what we normally observe in liquid helium-3. Although not rigorously tested, our best explanation of this phenomenon is that a turbulent foam of $l$ iquid and vapor develops in the experimental vessel as a result of our pulsed heating experiments. The 


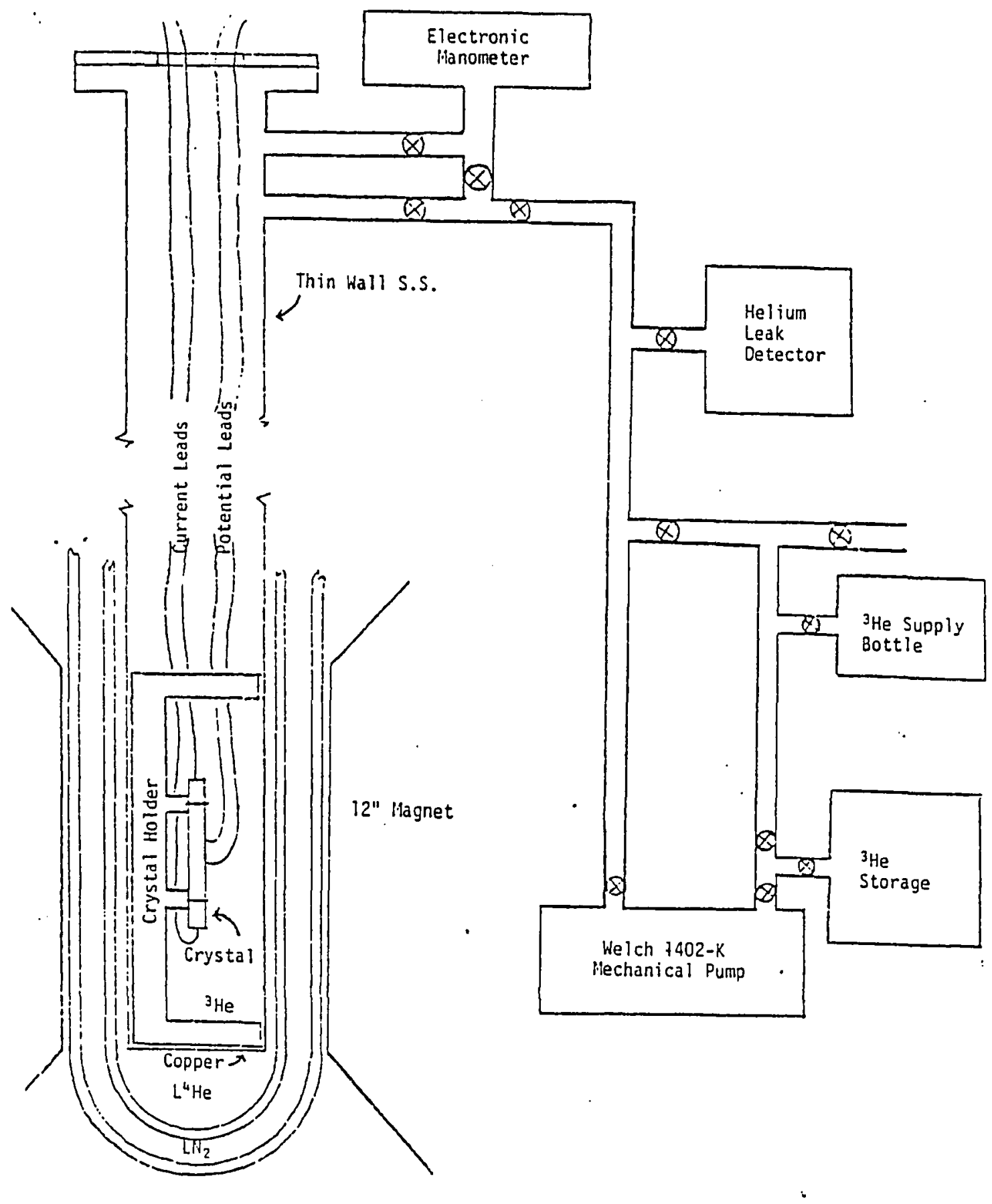
Figure 14 : Experimentàl apparatus with recycling for helium-
3 inner system. 
inhibited heat transfer does not revert to normal until a substantial amount of time has elapsed or the temperature of the 1 iquid helium- 4 bath is reduced resulting in condensation inside the helium-3 vessel. Because our pulsed experiments take place in the order of a few milliseconds and are limited to a penetration into the liquid helium bath of less than $0.1 \mathrm{~mm}$ (from $(\alpha t)^{1 / 2}$ ), we do not believe that this problem could be a substantial source of measurement error for the results reported here.

Equation 12 is a relatively complicated non-linear algebraic equation relating the nucleation rate to the 1 iquid temperature, pressure, and to the values of temperature and pressure dependent thermophysical properties. By setting the nucleation rate, $J$, equal to one critical nuclei per unit volume per unit time, we may separate the temperature and pressure dependent parts and solve easily and accurately for pressure as a function of homogeneous nucleation temperature. Although the choice of $\mathrm{J}=1$ is arbitrary, this rate is extremely sensitive to temperature and a change in temperature of 18 results in a change in the predicted nucleation rate by more than 10 orders of magnitude. The corresponding bath temperature may be found from the vapor pressure curve.

\section{EFFECT OF SURFACE TENSION}

For our numerical calculations we have used a vapor 
pressure temperature scale from sydoriac (1964), liquid and vapor densities from Kerr (1970), and surface tension values from Iino (1985). It should be noted that the surface tension data are very recent and differ enough from Zeno'veva's (1955) values used by Sinha et al to result in theoretical predictions of $\mathrm{T}_{\mathrm{h}}$ several percent lower than those published previously (Sinha 1982b). Thus our experimental values for $T_{h}$ in helium-3 do differ measureably from what was originally expected. To the extent that our experimental results can be used as a benchmark, it seems clear that Iino's helium-3 surface tension data are to be preferred to those of Zeno'veva.

\section{OBSERVATIONS}

In their measurements of the homogeneous nucleation temperature of helium-4, Sinha et al reported that the observed maximum superheat, $\mathrm{T}_{\max }$, remained constant over a wide range of applied heater powers (1982b). We have been unable to reproduce this observaton in either helium-3 or helium-4 but instead observe that $\mathrm{T}_{\max }$ increases slightly and $l$ inearly with increasing applied powers. Figure 15 is a graph of this measured maximum superheat temperature as a function of applied power. Although there are several possible factors that could contribute to the observed increase in maximum superheat with increasing heat flux our results suggest that the Kapitza resistance is the 


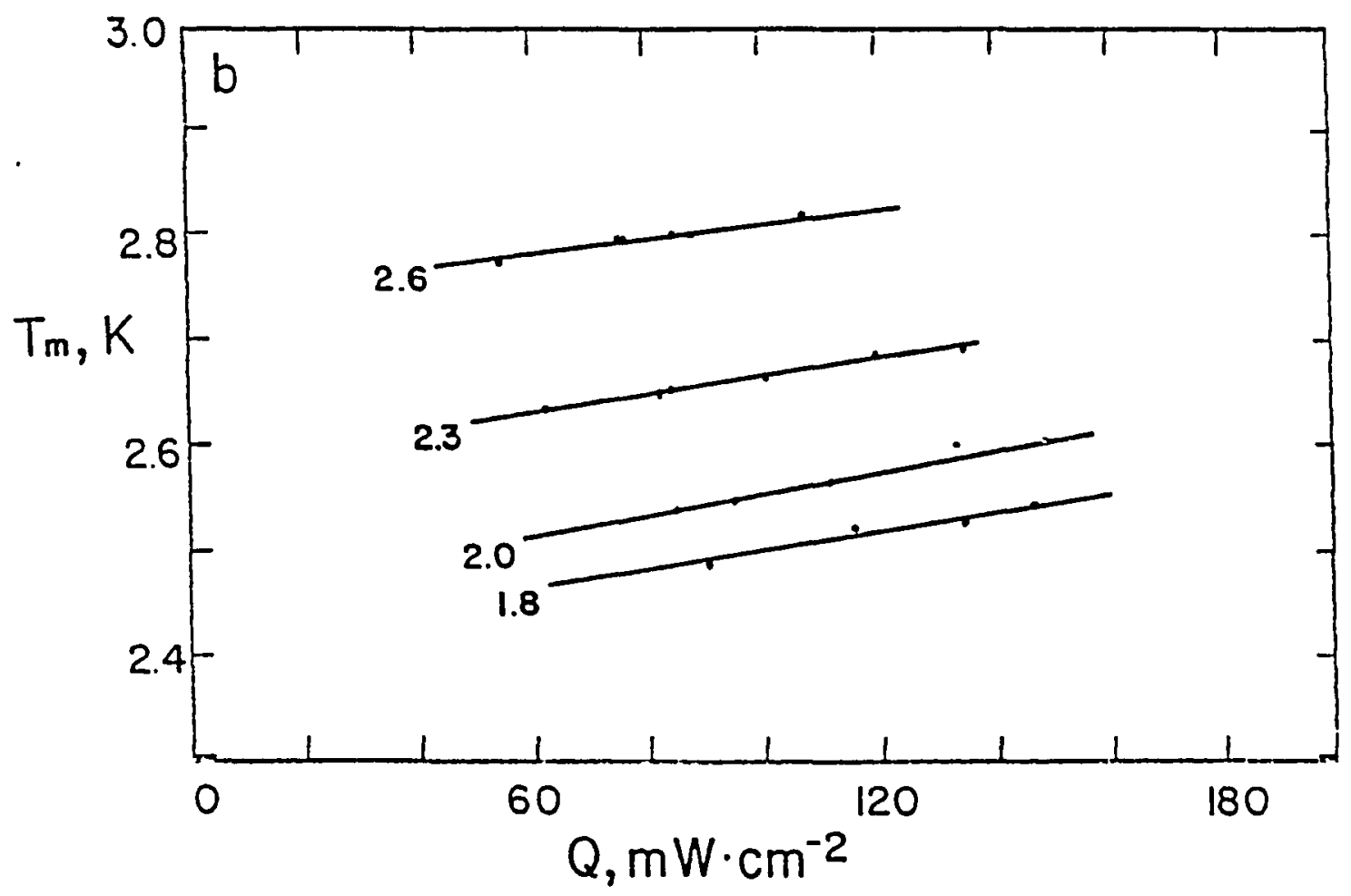

Figure 15: Measured maximum superheat vs heat flux at various bath temperatures. The data were taken in helium-3. 
overwhelming factor. (Cheeke 1973, Lezak in press) It is possible that the Sinha group's inability to observe the Kapitza $\Delta^{T}$ was due to a combination of factors including their less restricted geometry and higher crystal quality which allowed the use of relatively lower applied powers, an analog data acquisition and analysis system which may have made correction of errors due to non-ohmic effects more difficult than with our digital equipment, and the relativeIy lower Kapitza resistance at the higher temperatures afforded to helium-4 research.

Although our individual measurements fall somewhat above the predicted homogenoeus nucleation temperature for helium-3 we feel that the physically relevant quantity is the extrapolated zero-power intercept of a series of experiments taken at different powers. This analytic technique is expected to yield the actual values of liquid temperature without any influence of the Kapitza $\Delta^{\mathrm{T}}$. This approach is supported by the fact that the caclulated slopes of the $\Delta^{T}$ vs power curves are close to those expected from a prediction based on Kapitza thermal boundary resistance calculations.

Our experimental results are shown in Fig. 16 which is a reduced pressure vs reduced temperature graph. It is similar to Fig. 13 but has more detail in the regions of interest. We have included it for increased clarity. The helium-3 data points shown are act:ally the extrapolated 


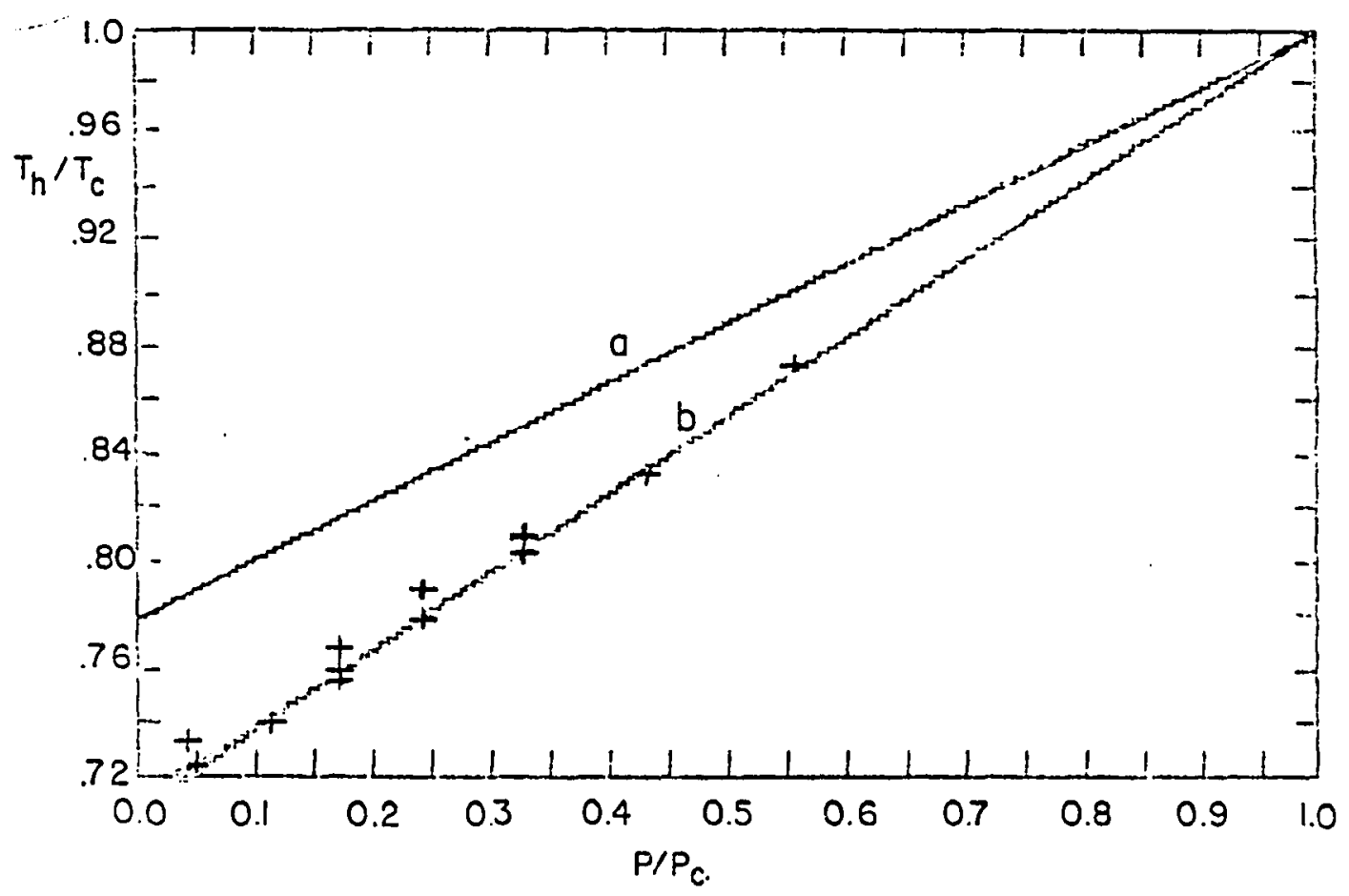

Figure 16: Scaled homogeneous nucleation temperature vs scaled pressure. This figure is similar to Fig. 13 except the experimental results reported in this paper have been emphasized. 
zero-power intercepts of a large number of individual transient superheating experiments. It can be seen that these results are close to the theoretical prediction based on Eq.12. The slight scatter in our results is discussed in the next section. This confirmation of homogeneous nucleation theory is consistent with results in helium-4 previously reported by Nishigaki and Saji (1983) in a quasisteady state experiment as well as with the transient superheating results reported by Sinha et al (1982b).

\section{ERROR ANALYSIS}

We have seen that, within a relatively small margin of error, the classical nucleation theory is shown to hold for predictions of thermally activated liquid to vapor homogeneous nucleation in helium-3. These measurements have thus extended the known range of applicability of this theory to the extreme limits of quantum liquids at positive pressures. Our experimental results were subject to both random and systematic errors. The relevant quantity in the analysis of random errors is the standard deviation in the extrapolated zero-power intercepts. This was calculated for each extrapolation and was typically in the range of 20-40 milli-Kelvin, a figure which is consistent with our expectations based on analysis of the experimental apparatus, associated electronics, and bath temperature uncertainty (Brodie 1981). 
Although our results are consistent with present theory, there are a number of factors that could systematically influence the experimental results. Nishigaki and Saji (1983) have suggested that the slight discrepancy in their quasi-steady state measurements with homogeneous nucleation theory could be due to early and spontaneous bubble formation as predictea by fluctuation nucleation theory (Hirth 1963). Sinha et al had suggested that because of the relatively small superheated layer and transient nature of their experiment, which is similar to ours, such spontaneous nucleation, as well as heterogeneous nucleation, should be negligible (1982b). A factor which could result in measured values lowe: thain expected is the heat conduction from the bismuth crystal heater-thermometer along the copper resistance sensing leads into the non-superheated liquid helium bath a short distance away (Hennecke 1970, Singh 1976, Keltner 1981). We have so far been unable to calculate precisely the expected magnitude of this error but results of experiments with differing wire and crystal sizes, which would be expected to result in differing relative errors, suggest that this factor may be safely neglected in our fast transient experiments.

\section{DISCUSSION}

The results reported above represent a verification of Becker-Doring classical nucleation theory in the most 
strongly quantum liquid, as measured by its de Boer parameter, and the completion of the positive pressure phase of a more extensive program of mapping out the entire boundary of the metastable region of liquid helium-3.

These measurements were made in the range of approximately 2.4 to $2.8 \mathrm{~K}$ and correspond to a bath temperature range of about 1.4 to $2.6 \mathrm{~K}$. Previous transient superheating work in helium-4 was limited by the $x$ transition at $2.18 \mathrm{~K}$ which corresponds to a homogeneous nucleation temperature of about $4.0 \mathrm{~K}$ (Sinha 1982b). In order to extend our measurements to still lower temperatures it will be necessary to move into the negative pressure regime with a tensile strength experiment. Our motivations for this are twofold. First, it is desireable to extend the range of verification of the classical nucleation theory for the liquid to vapor phase transition to as low a temperature as possible. In particular, we would like to go below the limit of about $0.8 \mathrm{~K}$ to which the general theory has been tested; in this case in helium-3/helium-4 phase separation experiments by Sinha and Hoffer (1983). Second, we are approaching the temperature, $1 *$, at which the rate of classical thermally activated nucleation of critical sized vapor nuclei over the energetic barrier to formation is predicted to equal that of quantum subbarrier tunnelling transitions (Lifshitz 1972, Akulichev 1975). This temperature has been predicted to be about $0.2 \mathrm{~K}$ for helium- 
3 and about $0.3 \mathrm{~K}$ for helium-4. If observed, this quantum transition will represent the first known regime in which classical nucleation theory is incomplete and the first observation of this particular nucleation mechanism.

It is of interest to note, however, that quantum tunnelling nucleation has been predicted to occur in other circumstances. In particular, Coleman (1980) and others (Guth 1981, Steinhardt 1982) have suggested that during the formation of the early universe, Higgs fields could supercool far below the $10^{27} \mathrm{~K}$ at which symmetry-breaking phase transitions are predicted to occur. The resulting metastable false vacuum would have a large negative pressure, as in the case of our tensile strength nucleation, and would eventually collapse because the Higgs field in a small region of space would tunnel through the energy barrier and nucleate a bubble of the broken-symmetry phase. It is interesting that an experiment at about $10^{\circ} \mathrm{K}$ could have much to say about the physics of the early universe at $10^{27} \mathrm{~K}$ some $10^{-34}$ seconds after its inital formation. 
REFERBACES

Adler, J.G. and T.A. Will, "Instabilities in superconducting Tunnel Junctions in Different Thermal Environments," App. Phys. Lett. 42,904 (1983).

Akulichev, V.A. and V.A. Bulanov, "Strength of Quantum Liquids," Sov. Phys. Acoust. 20, 501 (1975).

Albrecht, A. and P.J. "Steinhardt, "Cosmology for Grand Unified Theories with Radiatively Induced Symmetry Breaking," Physical Review Letters 48, 1220 (1982).

Apfel, R.E. "A Novel Technique for Measuring the Strength of Liquids," J. Acoust. Soc. Am. 49, 145 (1971).

Apfel, R.E. "Acoustic Cavitation," Methods of Experimental Physics 19, 355 (1981).

Baidakov, V.G., A.M. Kaverin and V.P. Skripov, "Acoustic Cavitation in a Highly Superheated Liquid," Sov. Phys. Acoust. (USA) 27,387 (1981).

Beams, J.W. "Tensile Strength of Liquid Helium II." Phys. Rev. 104,880 (1956).

Beams, J.W. "Tensile Strengths of Liquid Argon, Helium, Nitrogen, and Oxygen," Phys. Fluids 2, 1 (1959).

Becker, R. and $w$. Doring, "The Kinetic Treatment of Nuclear Formation in Supersaturated Vapors," Ann. Phys. 24, 719 (1935).

Bennemann, K.M. and J.B. Ketterson, The Physics of Liquid and Solid Helium, Part I. John Wiley and Sons, New York $(1967)$.

Blander, M. and J.L. Katz, "Bubble Nucleation in Liquids," AIChE J. 21, 833 (1975).

Bewilogua, L. and C. Gladin, "Condensed Rare Gases," Contemp. Phys. 9, 277 (1968).

Birkhoff, G., R.S. Margulies and W.A. Horning, Physics of Fluids 1, 201 (1958). 
Brodie, L.C., D.N. Sinha, J.S. Semura, and C.E. Sanford, "Transient Heat Transfer into Liquid Helium I," J. App I Phys. 48, 2882 (1977).

Brodie, L.C., D.N. Sinha, C.E. Sanford and J.S. Semura, "Bismuth Magnetoresistive Thermometry for Transient Temperature Measurements in Liquid Helium," 99 th ASME Winter Annual Meeting, San Francisco (1978) 78-WA/HT-4.

Brodie, L.C., D.N. Sinha, C.E. Sanford and J.S. Semura, "Bismuth Magnetoresistive Thermometry for Transient Temperature Measurements in Liquid Helium," Rev. Sci. Instr. 52, 1697 (1981).

Butler, A.P., G.B. James, J. Maddock and W.T. Norris, "Improved Pool Boiling Heat Transfer to Helium from Treated Surfaces and its Application to superconducting Magnets," Int. J. Heat Mass Transfer, 13, 105 (1970).

Cardona, M. and I, Ley, Photoemission in Solids, Topics in Applied Physics, Vol. 27. Springer-Verlag (1978).

Cheeke, J.D.N., B. Herbal, J. Richard and R.R. Turkington, "Kapitza Resistance of Low Acoustic Impedence Solids (Ice and Bismuth)," Physics Letters 46A, 81 (1973).

Coleman, S. and F. Deluccia, " Gravitational Effects on and of Vacuum Decay," Phys. Rev. D 21, 3305 (1980).

Finch, R.D., Taylor, G.J. Wang. R. Kagiwada, M. Barmatz, and Isadore Rudnick, "Studies of the Threshold-ofCavitation Noise in Iiquid Helium," J. Acoust. Soc. Am. $40,211(1966)$.

Giarratano, P.J. and W.G. Steward, "Transient Forced Convection Heat Transfer to Helium During a step in Heat Flux," Journal of Heat Transfer-Trans ASME 105, 350 (1983).

Guth, A.H., "Inflationary Universe: A Possible Solution to the Horizon and Flatness Problems," Physical Review D, 23,347 (1981)

Hennecke, D.K. and E.M. Sparrow, "Local Heat Sink on a Convectively Cooled Surface-Application to Temperature Measurement Error," Int. J. Heat Mass Transfer 13, 287 $(1970)$.

J.P. Hirth, J.P. and G.M. Pound: Progress in Materials Science (Pergamon Press, 1963) Vol. 11, p. 149 and references cited therein. 
Hord, J., R.B. Jacobs, C.C. Robinson, and L.L. Sparks," Nucleation Characteristics of Static Liquid Nitrogen and Liquid Hydrogen," Journal of Engineering for Power, Trans. ASME A86, 485 (1964).

I ino, M., M. Suzuki, A.J. Ikushima, and Y. Okuda, "Surface Tension of Liquid $3-\mathrm{He}$ down to $0.3 \mathrm{~K}, "$ Journal of Low Temperature Physics, 59, 291 (1985) (tentative).

Katz, J.I., C.J. Scoppa, N.B. Kumar and P. Mirabel, "Condensation of a Supersaturated Vapor," J. Chem. Phys. 63, 448 (1975).

Kaverin, A.M., V.G. Baidakov, and V.P. Skripov, "Spontaneous Nucleation Frequency in Superheated Liquid Xenon and Krypton," J. Eñg. Phys. (USA) 38, 408 (1980).

Keltner, N.R. and J. V. Beck, "Ünsteady Surface Element Method," Journal of Heat Transfer, Trans. ASME series C. 103,759 (1981).

Kerr, E.C. and R.H. Sherman, "The Molar Polarizability of ${ }^{3}$ He at Low Temperatures and its Density Dependence," J. Low Temp. Physics 3,451 (1970).

Kim, Y.I., Li-He Lin, C. Chuang, and T.H.K. Frederking, " Influence of Helium I Layer Formation on the CopperHelium-4 Kapitza Resistance at Helium II Bath Temperatures," App. Phys. Lett. 43, 451 (1983).

Kosky, P.G. and D.N. Lyon, "Pool Boiling Heat Transfer to Cryogenic Liquids," A.I. Ch. E. J. 14, 372 (1968).

Lezak, D., L.C. Brodie and J.S. Semura, "Photographic Studies of Light Induced Nucleation of Boiling at the interface of a Solid and Superheated Liquid Helium I," Cryogenics 23, 659 (1983).

Lezak, D., I.C. Brodie and J.S. Semura, "Light Induced Nucleation of Vapor Bubbles at the Interface of asolid and Superheated Liquid Helium I: Test of the Photoelectron Hypothesis," Cryogenics 24, 211 (1984a).

Lezak, D., L.C. Brodie and J.S. Semura, "Light Induced Cooling of a Heated Solid Immersed in Liquid Helium I," Advances in Cryogenic Engineering 29, 289 (1984b).

Lezak, D., L.C. Brodie, and J.S. Semura, "Measurement of the Kapitza Thermal Boundary Conductance from Bismuth to Normal Liquid Helium Isotopes 3 and 4 at their Homogeneous Nucleation Temperatures," to be published. 
Lifshitz, I.M. and Yu.: Kagan, "Quantum Kinetics of Phase Transitions at Temperatures Close to Absolute Zero," Sov. Physics-JETP 35, 206 (1972).

Marston, P.I., "Tensile Strength and Visible Ultrasonic Cavitation of Superfluid 4-He," J. Low Temp. Phys. 25, $383(1976)$.

MCConnel1, P.M., M. L. Chu Jr., and R.D. Finch, "Mechanism of Ultrasonic Cavitation Nucleation in Liquid Helium by Quantized Vortices," Phys. Rev. A 1, 411 (1970).

Metzger, W., R.P. Huebener, and K.P. Selig, "Transient Solid-Iiquid He Heat Transfer and onset of Film Boiling," Cryogenics 22, 387 (1982).

Mikic, B.B., W.M. Rohsenow and P. Griffith, "On Bubble Growth Rates," Int J. Heat Mass Trans. 13, 657 (1970).

Nishigaki, K. and Y. Saji, "Superheat Limit of 4-He and Its Quantum Deviation from Classical Behavior," Journal of the Physical Society of Japan 52, 2293 (1983).

Northby, J.A. and T.M. Sanders Jr., "Photoejection of Electrons from Bubble States in Liquid Helium," Phys. Rev. Lett. 18, 184 (1967).

Pavlov, Y.M., Potekhin, S.A. and V.A. Shugayev, "Study of the Mechanism of Helium Boiling with the Aid of High Speed Filming," Heat Transfer-Soviet Research 12, 45 (1980).

Plesset, M.S. and S.A. Zwick, J. Appl. Phys. 25, 493 (1954).

Reeber, M.D., "Heat Transfer to Boiling Helium," J. Appl. Phys 34, 481 (1963).

Schmidt, C. "Transient Heat Transfer to Liquid Helium and Temperature Measurement with a Response Time in the Microsecond Region," Appl. Phys. Lett. 32, 827 (1978).

Singh, B.S. and A. Dibbs, "Error in Temperature Measurement Due to Conduction Along the Sensor Leads," Journal of Heat Transfer, Trans. ASME serics C. 98, 491 (1976).

Sinha, D.N., I.C. Brodie, J.S. Semura and F.M. Young, "Premature Transition to stable Film Boiling Initiated by Power Transients in Liquid Nitrogen," Cryogenics 19, $225(1979)$.

Sinha, D.N., PhD. Thesis, Portland State University (1980). 
Sinha, D.N., L.C. Brodie, J.S. Semura and D. Lezak, "Light Induced. Enhancement of Transient Heat Transfer From a Solid into Liquid Helium I," Cryogenics 22, 271 (1982a).

Sinha, D.N., J.S. Semura and I.C. Brodie, "Homogeneous Nucleation in 4-He: A Corresponding-states Analysis," Phys. Rev. A 26, 1048 (1982b).

Sinha, D.N., J.S. Semura, and L.C. Brodie, "Quantum Effects on the Temperature Dependence of Surface Tension of Simple Liquids," J. Chem. Phys. 76(4), 2028 (1982C).

Sinha, D.N., J.S. Semura and L.C. Brodie, "Superheating Limits of Liquid Helium I," Cryogenics 22, 391 (1982d).

Sinha, D.N. and J.K. Hoffer, "Tricritical Slowing Down of Phase Separation in Liquid 3-He-4-He Mixtures," Phys. Rev. Lett. $50(7), 515$ (1983).

Skripov, V.P., V.G. Baidakov, and A.M. Kaverin, "Nucleation in Superheated Argon, Krypton, and Xenon Liquids," Physica 95A, 169 (1979).

Skripov, V.P., Metastable Liquids, John Wiley \& Sons, New York (1974).

Smith, C.W., G.F. Burke and I.A. Rooney, "Experiments Involving Sonification of Liquid Helium at $4.2 \mathrm{~K}$ in the Cathode-Anode Interspace of a Photodiode Tube," J. Acoust. Soc. Am. 60,378 (1976).

Snyder, N.S., "Thermal Conductance at the Interface of a Solid and Helium II," NBS Technical Note 385 , December, 1969 .

Steinhardt, P.J. and A. Albrecht, "Cosmology for Grand Unified Theory with Radiatively Induced Symmetry Breaking," Phys. Rev. Lett. 48, 1220 (1982).

Steward, W.G. "Transient Heat Transfer Phase I-Static Coolant," Int. J. Heat Mass Transfer 21, 863 (1978).

Sydoriac, S.G., T.R. Roberts and R.H. Sherman, "1962 ${ }^{3} \mathrm{He}$ Scale of Temperatures," J. Res. Nat. Bur. Std. 68A, 547 (1964).

Touloukian, Y.S., Thermophysical Properties of Matter, 1, 25 (1970).

Touloukian, Y.S., "Master Index to Materials and Properties," IFI/Plenum Press, 1979. 
Tong, L.S. "Boiling Heat Transfer and Two-Phase Flow," Robert E. Krieger Publishing Company, Huntington, N.Y. (1975).

Tsukamoto, O. and S. Kobayashi, "Transient Heat Transfer Characteristics of Liquid Helium," Journal of Applied Physics 46, 1359 (1975).

Volmer, M. and A. Weber, "Nucleus Formation in Supersaturated Systems," Z. Phys Chem. 119, 277 (1926).

Weast, R.C.,Ed. CRC Handbook of Chemistry and Physics, CRC Press $1982,63 \mathrm{rd}, \mathrm{Ed} . \mathrm{E}-78$.

Woolf, M.A. and G.W. Rayfield, "Energy of Negative Ions in Liquid Helium by Photoelectric Injection," Phys. Rev. Lett. $1315,235(1965)$.

Zeno'veva, K.N., Sov. Physics JETP 1,173 (1955). 


\section{APPENDIX A}

\section{PREPARATION OF BISMUTH SINGLE CRYSTALS FOR USE IN}

MAGNETORESISTIVE THERMOMETRY IN LIQUID HELIUM

Most of the results reported in this dissertation have resulted from research with bismuth crystal magnetoresistive heater-thermometers in liquid helium. These devices have a unique combination of properties which make them almost ideally suited for accurate fast transient thermometry at liquid helium temperatures. The principal drawbacks of these devices for thermometric applications are related to the fact that the temperature sensitivity, 1/R (dR/dT), is greatly reduced by impurities or imperfections in the crystal structure. For any given application both the accuracy and signai to noise fatio of the thermometer are inevitably influenced by this sensitivity and, in the event of a relatively poor crystal, the actual usability can be adversely compromised. For this reason, careful attention must be paid to the optimization of crystal quality at each step of the manufacturing process.

There are, naturally, a number of steps in getting from pure $(0.99999$ - 0.999999) polycrystalline bismuth to an actual thermometric measurement. Some of these are, in rough order: 


\section{CRYSTAL GROWTH}

1) Growth of an appropriate seed crystal.

2) Casting of a polycrystalline slug with appropriate dimensions for the application at hand.

3) Growth of a single crystal with an appropriate crystal lographic orientation from the seed and slug.

4) Purification by repeated zone melting of the crystal.

The crystals are grown in a rough vacuum in a mold constructed from vycor plates lubricated with carbon black (from a candle). The bismuth is from cerro de Pasco 159 purity) or cominco (69 purity). The bismuth is melted by a zone melting apparatus in which a clock motor moves a heater coil along at about $1 / 2 \mathrm{~mm}$ per minute. We have used two furnaces, one for the large and another for the small sized crystals.

CRYSTAL MOUNTING

5) Mounting the crystal in an appropriate holder with a minimum of stress and damage.

6) Attachment of appropriate current supply and voltage sensing leads with adequate physical strength and a minimum of stress and damage.

7) Determination of surface area between lead pairs (for calculations of power and heat flux). 
8) Transfer of the crystal/holder combination from the workbench to an appropriate cryomagnetic dewar system. Before mounting, the orientation of each crystal is determined to within about one degree by means of an optical goniometer. The crystals used in the work reported here had their trigonal axes perpendicular to the face with a binary axis perpendicular to the length.

The large crystals are about $2 \mathrm{~mm}$ thick, $5 \mathrm{~mm}$ wide, and average about $6 \mathrm{~cm}$ long. The small crystals are about $2 \mathrm{~mm}$ by $2 \mathrm{~mm}$ and about $4 \mathrm{~cm}$ in length. They are mounted on a variety of holders designed to maximize mechanical stability and exposure to the liquid helium and to our light sources when relevant for 1 ight effect studies. Number 32 copper wires are soldered with bismuth to either end of each crystal to serve as current leads and as many as six leads of number 40 - 44 copper wire are spot welded or soldered along the crystal for potential leads. Glue and light thread are used to hold the crystal in place.

\section{CALIBRATION}

9) Determination of crystal lographic orientation by rotating the magnetic field.

10) Determination of sensitivity at various fields and bath temperatures.

11) Quasi-static calibration of resistance vs temperature (typically against a vapor pressure curve). 
Calibration for $\Delta^{R}$ vs $\Delta^{T}$ is relatively straightforward after the desired magnetic field and orientation have been determined. We have used a relatively low current 4terminal ohmmeter and did not correct for any possible nonohmic effect at this stage of the experiment.

\section{EXPERIMENT AND DATA ANALYSIS}

12) Dynamic experimental measurements to find $\Delta^{R(t)}$ with appropriate correction for any nonohmic effects.

13) Conversion from $\Delta^{R(t)}$ to $\Delta^{T(t)}$.

In the work reported here we used a 4-terminal resistance measuring network in which the heating current served to provide the requisite potential drop. Correction for the non-ohmic effect is discussed below. 


\begin{abstract}
APPENDIX B
USE OF GERMANIUM CRYSTALS AND RHODIUM-IRON WIRE FOR FAST TRANSIENT THERMOMETRY IN LIQUID HELIUM
\end{abstract}

There are a number of properties which a thermometer must have in order to be an adequate fast transient thermometer in liquid helium. Principal among these are low thermal mass and high thermal diffusivity and conductivity. These are important to ensure that the thermometer causes as little perturbation to the liquid helium as possible and that there is thermal equilibrium within the thermometer itself. In addition, the applications reported in this dissertation have all used our thermometric elements as heater-thermometers where the electric current which enables us to measure the resistance of these elements is set high enough that it also generates substantial amounts of joule heating.

of the three different types of thermometers that we have used in our research, two have the advantage of being commercially available: the rhodium-iron wire and the doped germanium crystal were both purchased from Lake Shore Cryotronics, Westerville, Ohio 43081. The germanium crystal had the additional advantage of coming with leads already attached at the factory, which saved a great deal of trouble 
for our $1 \mathrm{ab}$ and minimized the risk of damage during the mounting process.

In each of these devices, the thermometric property which was exploited for our temperature measurements was the sensitivity of the electrical resistance of the device to changes in temperature and the actual differential electronic technique was quite similar from one device to another. For purposes of comparison, we have tabulated a number of relevant properties of these devices in Table 1. We can see that the germanium crystal suffers from a relatively low thermal conductivity and is thus unsuitable for very fast experiments. The rhodium-iron wire, while extremely fast, may be a bit too small to be useful in cases where the approximation of semi-infinite planar geometry is required. In this case, increasing wire size beyond some limit will decrease the resistance to the point that the current becomes unmanageably high for the generation of joule heating at an adequate rate. None-the-less, we have observed the light effect in each of these devices and have made crude measurements of the homogeneous nucleation temperature. 
TABLE I

\section{Bismuth}

Size (mm)

$2 \times 2$

Thermal

Conductivity

$\left(\mathrm{W} / \mathrm{Cm} \mathrm{K}^{*}\right)$

Thermal

Diffusivity

$\left(\mathrm{cm}^{2} / \mathrm{sec}\right)$

$3 \times 10^{3}$

$1 \times 10^{5}$

$7 \times 10^{3}$

Rhodium +

.05 dia.

12

8
Thermal Response

( $\mathrm{sec})$

Internal

$\left(i^{2} / x\right)$

$4 \times 10^{-6}$

$1 \times 10^{-9}$
External
$\left(\rho \vee\left(h_{k} /\right)\right.$

$$
10^{-4}
$$

Sensitivity

$1 / \mathrm{R}_{1}(\mathrm{dR} / \mathrm{dT})$

$\left(\mathrm{K}^{-1}\right)$. 1

Linearity
$3 \times 10^{-6}$

Data for this table, for $4 \mathrm{~K}$, have been taken from Touloukian (1970), Snyder (1969), and Cheeke (1973), as well as from our own experimental results. The results for the external time constant for Rhodium-Iron and Germanium should be considered to be order of magnitude estimates only. In particular, the value used for the thermal conductivity for Germanium is probably quite a bit higher than the actual value for our heavily doped sample. While the dopant is necessary to increase the conductivity of our sample it also acts to upset the lattice structure of the crystal and could result in a decrease in thermal conductivity of several orders of magnitude. In our experiments we observed that the germanium thermometers appeared to have thermal response times on the order of some fractions of a millisecond--about 100 times slower than reported above. 
APPENDIX C

CRYSTAL BALANCE TECHNIQUES FOR FAST TRANSIENT THERMOMETRY USING NON-OHMIC. RESISTIVE SENSORS

All of the quantitative results reported in this dissertation depend directly upon an accurate determination of the sensor resistance as a function of time. In principle, the sensor resistance $R(t)$ could be directly determined by evaluating the quantity $V(t) / I(t)$. This value could then be compared to some previously determined calibration curve relating the measured sensor resistance to steady-state sensor temperature and, in the case of a nonohmic sensor, to the applied current. In addition to the obvious Iimitation of temperature sensor response time there remain practical limits to measurement technology which render this entire approach inappropriate for our most sensitive measurements. Some of these are:

1) Limited resistance change resulting in a low value of $\Delta^{R / R}$ as well as a low signal to noise ratio, 2) Finite current rise time, 3) Distributed inductance and capacitance in the current loop,

4) Difficulties in establishing the $R, T, I$ surface for calibration in the presence of a large non-ohmic 
effect.

In order to overcome the limitations imposed by the first restraint, we utilize a differential measurement method in which the quantity $\Delta R(T, I)$ is determined rather than $R(T, I)$. This circuit is outlined in Fig. 17. The advantage of this approach is obvious if we put in in the perspective of a typical measurement. For ${ }^{3}$ He at a bath temperature of $2.5 \mathrm{~K}$ the expected homogeneous nucleation temperature is $2.77 \mathrm{~K}$. Typical crystal sensitivity, $\mathrm{S}=$ $\left(1 / R^{\star} d R / d T\right)$ will be smaller than -0.1 and typical crystal resistance will be about 10 ohms. With a current of $100 \mathrm{ma}$, a voltage of $\mathrm{V}=\mathrm{I} * \mathrm{R}=1.0$ volts will be measured between the sensor leads. At $2.77 \mathrm{~K}$ the crystal resistance will be $R^{*}\left(1+\Delta^{T *}\right)=9.73$ ohms and the measured voltage (assuming a constant current of $100 \mathrm{ma}$ ) will be 0.973 volts. In other words, the total resistance change is about $2.7 \%$ so our voltage and current measurements must be accurate to about $0.14 \%$ for a net $5 \%$ accuracy in the determination of the change in temperature. A differental measurement, on the other hand, need only be accurate to 5 for fhat same net result.

The differential technique, while solving the major problem of extracting the signal due to a change in temperature from the substantial steady voltage, introduces several additional problems. The most substantial of these relate to the necessity of accurately subtracting that 


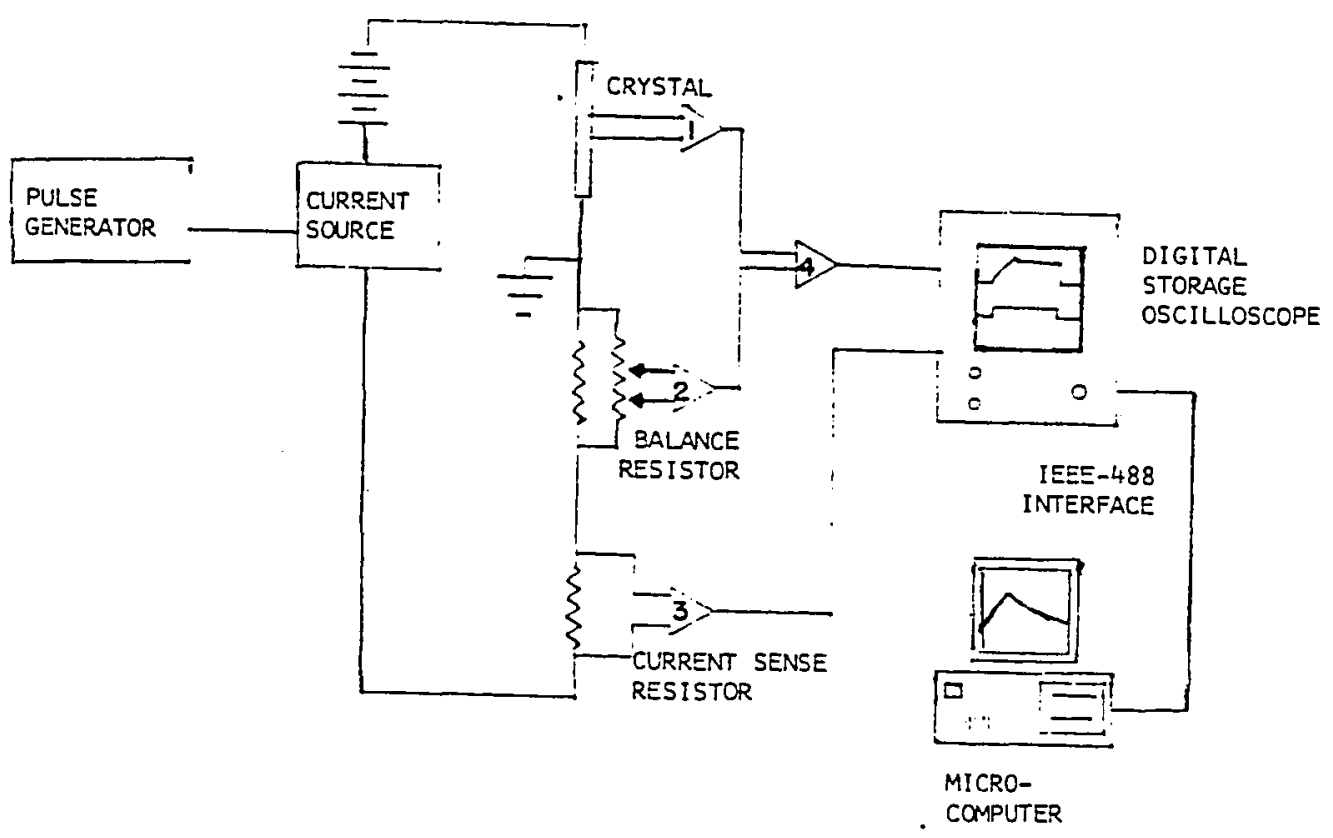

Figure 17: Schematic circuit diagram. 
portion of the signal that results from the value of the sensor resistance at the original temperature. Using our same example, a net accuracy of 58 requires that the differential voltage, which is 0.27 ohms times 100 ma equals 27 millivolts, be known to within about 1.4 millivolts-identical to the value established above. The consolation, of course, is the relative ease of establishing an accurate and steady balancing resistance compared to the difficulty of making a transient measurement with the same precision. Another difficulty of the differential technique has to do with the accurate establishment of the precisely correct balancing resistance. In the absence of substantial nonohmic behaviour, this can be rather easily accomplished using a pre-triggered oscilloscope and a relatively low current. This is shown in Fig. 19 a which has simplified drawings of typical oscilloscope traces at increasing currents. The electrical spikes that are present at the moment that the current is turned on, shown more clearly in Fig. 18, are due to transient non-linearities in the electrical curcuits and the finite rise time of the current pulse. As the current is increased the balance can be seen to remain the same although the actual balance becomes obscured at the highest currents due to the relative size and duration of the perturbation imposed by the sudden switching of the current pulse. A typical sensor with nonohmic behaviour is shown in Fig. $19 \underline{b}$. In this case, the 


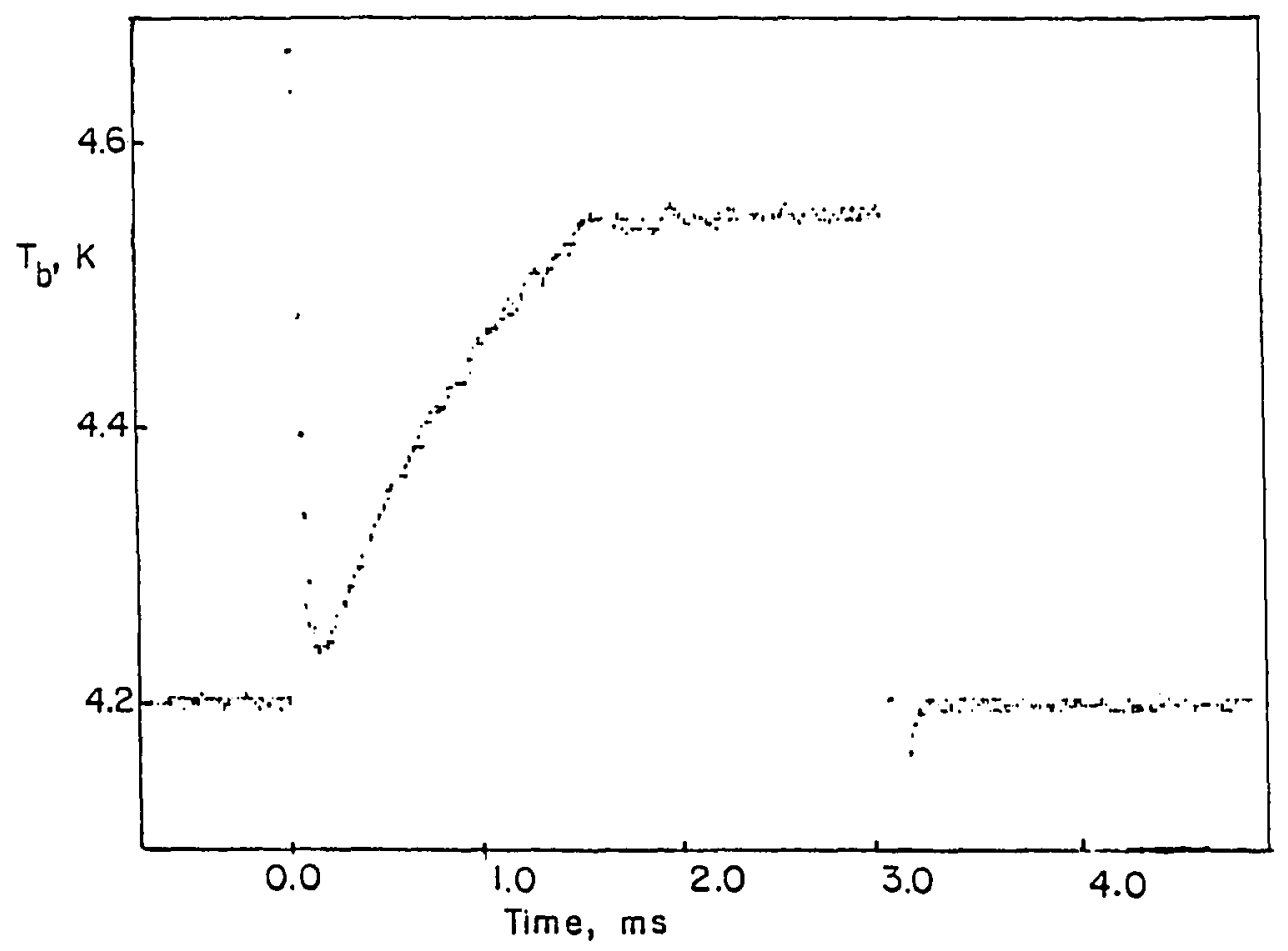

Figure 18: Typical medium quality crystal showing distinct spike at start of current pulse. The balance has been visualiy extrapolated to $t=0$. 

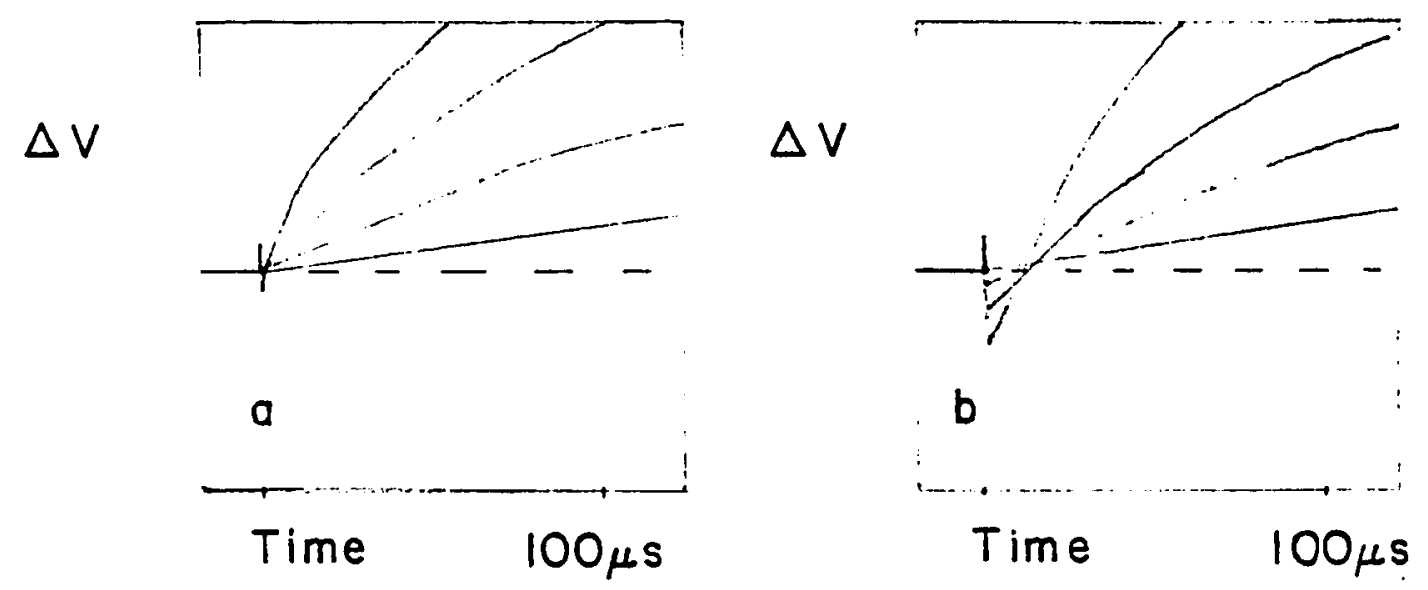

Figure 19: a) Balance curve for a crystal without non-ohmic behaviour. b) Balance curve for a crystal with nonohmic behaviour. The multiple curves on each figure demonstrate the effects of increasing the applied current without adjusting the crystal balance. 
crystal balance can be seen to shift from the low current value as the current is increased. In principle, the balance could be accurately recorrected at each current (thus giving the current dimension to the 3-dimensional $R, T, I$ surface) but in practice this is made difficult by the electrical noise and substantial spike at the start of the current pulse. Increasing the rate at which the current reaches its steady-state value can be helpful to a point but the distributed inductance in the circuit ensures that at least part of this turn-on noise increases as dI/dt. We have found that a rise time of about 10-30 us is usually the best compromise. 